\title{
Effectiveness of Chinese herbal medicine combined with Western medicine on deferring dialysis initiation for nondialysis chronic kidney disease stage 5 patients: a multicenter prospective nonrandomized controlled study
}

Yifan $\mathrm{Wu}^{1 \#} \wedge$, Chuang $\mathrm{Li}^{1,2 \#}$, Lei Zhang ${ }^{1,2}$, Chuan Zou ${ }^{1}$, Peng $\mathrm{Xu}^{1}$, Zehuai Wen ${ }^{3}$, Wenwei Ouyang ${ }^{3}$, Nizhi Yang ${ }^{1}$, Min Zhang ${ }^{4} \wedge$, Qizhan Lin ${ }^{1}$, Fuhua Lu ${ }^{1}$, Lixin Wang ${ }^{1}$, Kun Bao ${ }^{1}$, Daixin Zhao ${ }^{1}$, Lizhe Fu ${ }^{1}$, Xinfeng Guo ${ }^{5}$, Lihong Yang ${ }^{5}$, Aihua $\mathrm{Ou}^{6}$, Zehui $\mathrm{He}^{6}$, Heng Weng ${ }^{6}$, Jianmin $\mathrm{Li}^{7}$, Wei Shi ${ }^{8}$, Xiaoqin Wang ${ }^{9}$, Liqun Song $^{10}$, Yongli Zhan ${ }^{11}$, Wei Sun ${ }^{12}$, Lianbo Wei ${ }^{13}$, Niansong Wang ${ }^{14}$, Dingkun Gui ${ }^{14}$, Jihong Zhan ${ }^{15}$, Ying Lu ${ }^{16}$, Hongyu Chen ${ }^{17}$, Yuning Liu ${ }^{18}$, Hongtao Yang ${ }^{19}$, Ming Chen ${ }^{20}$, Yiping Wang ${ }^{21}$, Peiqing Zhang ${ }^{22}$, Yueyi Deng ${ }^{23}$, Lanfen Meng ${ }^{24}$, Xiaohong Cheng ${ }^{25}$, Feng Li ${ }^{26}$, Dajun $\mathrm{Yu}^{27}$, Damin Xu ${ }^{28}$, Jing'ai Fang ${ }^{29}$, Hongyan $\mathrm{Li}^{30}$, Junzhou $\mathrm{Fu}^{31}$, Yuansheng $\mathrm{Xie}^{32}$, Wenge $\mathrm{Li}^{33}$, Jinghong $\mathrm{Zhao}^{34}$, Yuanhang Huang ${ }^{35}$, Zhaoyu Lu ${ }^{1}$, Guobin Su ${ }^{1}$, La Zhang ${ }^{1}$, Xindong Qin ${ }^{1}$, Yuan Xu ${ }^{1}$, Yu Peng ${ }^{1}$, Haijing Hou ${ }^{1}$, Lili Deng ${ }^{1}$, Hui Liu ${ }^{1}$, Xina Jie ${ }^{1}$, Lichang Liu ${ }^{1}$, Fang Tang ${ }^{1}$, Hongfei Pei ${ }^{2}$, Ping Li $^{33}$, Wei Mao ${ }^{1,2}$, Xusheng Liu ${ }^{1,2,36 \wedge}$

${ }^{1}$ Department of Nephrology, Guangdong Provincial Hospital of Chinese Medicine (The Second Affiliated Hospital of Guangzhou University of Chinese Medicine), Guangzhou, China; ${ }^{2}$ State Key Laboratory of Dampness Syndrome of Chinese Medicine, Guangdong Provincial Hospital of Chinese Medicine (The Second Affiliated Hospital of Guangzhou University of Chinese Medicine), Guangzhou, China; Key Unit of Methodology in Clinical Research, Guangdong Provincial Hospital of Chinese Medicine (The Second Affiliated Hospital of Guangzhou University of Chinese Medicine), Guangzhou, China; ${ }^{4}$ The Second Affiliated Hospital of Guangzhou University of Chinese Medicine, Guangzhou, China; ${ }^{5}$ Evidence-based Medicine \& Clinical Research Service Group, Guangdong Provincial Hospital of Chinese Medicine(The Second Affiliated Hospital of Guangzhou University of Chinese Medicine), Guangdong Provincial Academy of Chinese Medical Sciences, Guangzhou, China; ${ }^{6}$ Department of Big Medical Data, Department of Clinical Epidemiology, Guangdong Provincial Hospital of Chinese Medicine (The Second Affiliated Hospital of Guangzhou University of Chinese Medicine), Guangzhou, China; ${ }^{7}$ Department of Spleen and Stomach Diseases, Guangdong Provincial Hospital of Chinese Medicine (The Second Affiliated Hospital of Guangzhou University of Chinese Medicine), Guangzhou, China; ${ }^{8}$ Department of Nephrology, First Affiliated Hospital of Guangxi University of Chinese Medicine, Nanning, China; ${ }^{9}$ Department of Nephrology, Hubei Provincial Hospital of Chinese Medicine, Wuhan, China; ${ }^{10}$ Department of Nephrology, First Affiliated Hospital of Heilongjiang University Of Chinese Medicine, Harbin, China; ${ }^{11}$ Department of Nephrology, Guang'anmen Hospital China Academy of Traditional Chinese Medicine, Beijing, China; ${ }^{12}$ Department of Nephrology, Jiangsu Provincial Hospital of Chinese Medicine, Nanjing, China; ${ }^{13}$ Department of Nephrology, TCM Integrated Hospital of Southern Medical University, Guangzhou, China; ${ }^{14}$ Department of Nephrology, The Sixth People's Hospital Affiliated to Shanghai Jiao Tong University, Shanghai, China; ${ }^{15}$ Department of Nephrology, First Affiliated Hospital of Guiyang College of Traditional Chinese Medicine, Guiyang, China; ${ }^{16}$ Department of Nephrology, Tong De Hospital, Zhejiang Province, Hangzhou, China; ${ }^{17}$ Department of Nephrology, Hangzhou Hospital of Chinese Medicine, Hangzhou, China; ${ }^{18}$ Department of Nephrology, Dongzhimen Hospital of Beijing University of Chinese Medicine, Beijing, China; ${ }^{19}$ Department of Nephrology, First Affiliated Hospital of Tianjin University Of Chinese Medicine, Tianjin, China; ${ }^{20}$ Department of Nephrology, Affiliated Hospital of Chengdu University of Traditional Chinese Medicine, Chengdu, China; ${ }^{21}$ Department of Nephrology, Anhui Provincial Hospital of Chinese Medicine, Hefei, China; ${ }^{22}$ Department of Nephrology, Heilongjiang Academy of Traditional Chinese Medicine, Harbin, China; ${ }^{23}$ Department of Nephrology, Longhua Hospital Affiliated to Shanghai University of Traditional Chinese Medicine, Shanghai, China; ${ }^{24}$ Department of Nephrology, Liuzhou Hospital of Traditional Chinese Medicine, Liuzhou, China; ${ }^{25}$ Department of Nephrology, Shaanxi Provincial Hospital of Chinese Medicine, Xi'an, China; ${ }^{26}$ Department of Nephrology, Xijing Hospital of The Fourth Military Medical University, Xi'an, China; ${ }^{27}$ Department of Nephrology, Xiyuan Hospital, Academy of Traditional Chinese Medicine, Beijing, China; ${ }^{28}$ Department of Nephrology, First Hospital of Peking University,

^ ORCID: Yifan Wu, 0000-0002-8498-8436; Min Zhang, 0000-0002-8470-7307; La Zhang, 0000-0002-8300-2890; Xina Jie, 0000-0003-3103-8813; Xusheng Liu, 0000-0002-0931-4925. 


\section{Page 2 of 18}

Wu et al. CHM on deferring dialysis initiation for stage 5 CKD patients

Beijing, China; ${ }^{29}$ Department of Nephrology, First hospital of Shanxi Medical University, Taiyuan, China; ${ }^{30}$ Department of Nephrology, Huadu District People's Hospital of Guangzhou, Guangzhou, China; ${ }^{31}$ Department of Nephrology, Guangzhou No.1 People's Hospital, Guangzhou, China; ${ }^{32}$ Department of Nephrology, China PLA General Hospital , Beijing, China; ${ }^{33}$ Department of Nephrology, China-Japan Friendship Hospital, Beijing, China; ${ }^{34}$ Department of Nephrology, Third Military Medical University Xinqiao Hospital, Chongqing, China; ${ }^{35}$ Department of Nephrology, General hospital of Guangzhou Military Command of PLA, Guangzhou, China; ${ }^{36}$ Guangdong Provincial Key Laboratory of Clinical Research on Traditional Chinese Medicine Syndrome, Guangdong Provincial Hospital of Chinese Medicine (The Second Affiliated Hospital of Guangzhou University of Chinese Medicine), Guangzhou, China

Contributions: (I) Conception and design: Y Wu, C Li, P Li, X Liu, W Mao, L Zhang, P Xu; (II) Administrative support: X Liu, W Mao, P Li; (III) Provision of study materials or patients: Y Wu, C Li, L Zhang, C Zou, P Xu, Q Lin, F Lu, L Wang, K Bao, D Zhao, N Yang, W Shi, X Wang, L Song, Y Zhan, W Sun, L Wei, N Wang, D Gui, J Zhan, Y Lu, H Chen, Y Liu, H Yang, M Chen, Y Wang, P Zhang, Y Deng, L Meng, X Cheng, F Li, D Yu, D Xu, J Fang, H Li, J Fu, Y Xie, W Li, J Zhao, Y Huang, Z Lu, G Su, L Zhang, X Qin, Y Xu, Y Peng, H Hou, L Fu, L Deng, H Liu, X Jie, L Liu, F Tang, P Li, W Mao, X Liu; (IV) Collection and assembly of data: Y Wu, J Li, M Zhang; (V) Data analysis and interpretation: Y Wu, M Zhang, J Li, W Ouyang, Z Wen, X Guo, L Yang, A Ou, Z He, W Mao, L Zhang, C Li; (VI) Manuscript writing: All authors; (VII) Final approval of manuscript: All authors.

\#These authors contributed equally to this work.

Correspondence to: Dr. Xusheng Liu, MD. State Key Laboratory of Dampness Syndrome of Chinese Medicine \& Guangdong Provincial Key Laboratory of Clinical Research on Traditional Chinese Medicine Syndrome \& Department of Nephrology, Guangdong Provincial Hospital of Chinese Medicine (The Second Affiliated Hospital of Guangzhou University of Chinese Medicine), no.111, Dade Road, Yuexiu District, Guangzhou 510120, China. Email: liuxusheng@gzucm.edu.cn; Dr. Wei Mao, PhD. State Key Laboratory of Dampness Syndrome of Chinese Medicine \& Department of Nephrology, Guangdong Provincial Hospital of Chinese Medicine (The Second Affiliated Hospital of Guangzhou University of Chinese Medicine), no.111, Dade Road, Yuexiu District, Guangzhou 510120, China. Email: maowei@gzucm.edu.cn; Dr. Ping Li, PhD. Department of Nephrology, China-Japan Friendship Hospital, Yinghua Donglu, Hepingli, Chaoyang District, Beijing 100029, China. Email: lp8675@163.com.

Background: In clinical practice, Chinese herbal medicine (CHM) purportedly has beneficial therapeutic effects for chronic kidney disease (CKD), which include delaying disease progression and dialysis initiation. However, there is a lack of high-quality evidence-based results to support this. Therefore, this study aimed to evaluate the efficacy of CHM combined with Western medicine in the treatment of stage 5 CKD.

Methods: This was a prospective nonrandomized controlled study. Stage 5 CKD (nondialysis) patients were recruited form 29 AAA class hospitals across China from July 2014 to April 2019. According to doctors' advice and the patients' wishes, patients were assigned to the CHM group (Western medicine + CHM) and the non-CHM group (Western medicine). Patient demographic data, primary disease, blood pressure, Chinese and Western medical drugs, clinical test results, and time of dialysis initiation were collected during follow-up.

Results: A total of 908 patients were recruited in this study, and 814 patients were finally included for further analysis, including 747 patients in the CHM group and 67 patients in the non-CHM group. 482 patients in the CHM group and 52 patients in the non-CHM group initiated dialysis. The median time of initiating dialysis was $9(7.90,10.10)$ and $3(0.98,5.02)$ months in the CHM group and non-CHM group, respectively. The multivariate Cox regression analysis showed that patients in the CHM group had a significantly lower risk of dialysis [adjusted hazard ratio (aHR): 0.38; 95\% confidence interval (CI): 0.28, 0.53] compared to those in the non-CHM group. After 1:2 matching, the outcomes of 160 patients were analyzed. The multivariate Cox regression analysis showed that patients in the CHM group had a significantly lower risk of dialysis (aHR: 0.32; 95\% CI: 0.21, 0.48) compared to patients in the non-CHM group. Also, the Kaplan-Meier analysis demonstrated that the cumulative incidence of dialysis in the CHM group was significantly lower than that in the non-CHM group (log-rank test, $\mathrm{P}<0.001$ ) before and after matching.

Conclusions: This study suggest that the combination of CHM and Western medicine could effectively reduce the incidence of dialysis and delay the time of dialysis initiation in stage 5 CKD patients.

Keywords: Chinese herbal medicine (CHM); stage 5 chronic kidney disease (stage 5 CKD); prospective 
nonrandomized controlled study; multicenter, dialysis

Submitted Dec 11, 2020. Accepted for publication Mar 20, 2021.

doi: $10.21037 / \mathrm{atm}-21-871$

View this article at: http://dx.doi.org/10.21037/atm-21-871

\section{Introduction}

Chronic kidney disease (CKD) is a serious disease which endangers human health and has become a major public health problem in the 21 st century. It has a high prevalence (1), leads to immense medical expenditure, is often complicated by cardiovascular disease, and therefore results in high disability and high mortality (2).

Stage $5 \mathrm{CKD}$ is the late-stage of this disease (3). Most patients have obvious clinical symptoms; however, some patients remain in a stable state (nondialysis) for a certain period of time after active treatment, thereby delaying the onset of dialysis. Stage 5 CKD without dialysis is an independent stage of CKD, and has been classified by the 10th revision of the International Classification of Diseases (ICD-10) as code N18.5. This means that there are no indications of dialysis in stage $5 \mathrm{CKD}$, and its condition can be temporarily stable or can be controlled by medicine, only requiring close monitoring (4). As for the choice of dialysis initiation timing, a large multicenter clinical study showed that there was no difference in the incidence of endpoints and adverse events between early initiation of dialysis and late initiation of dialysis in patients with stage 5 CKD (5). Another retrospective study with a large sample size showed that the risk of death after dialysis was positively correlated with the baseline glomerular filtration rate (GFR, greater than $5 \mathrm{~mL} / \mathrm{min} / 1.73 \mathrm{~m}^{2}$ ) at the start of dialysis, concluding that early initiation of dialysis may be harmful (6). In addition, the European Renal Best Practice (ERBP) guidelines have also pointed out that the timing of dialysis initiation should be carefully considered, and starting dialysis prematurely should be avoided (4). Therefore, conservative treatment (nonreplacement therapy) plays a crucial role in the late stages of CKD.

In clinical practice, Chinese herbal medicine (CHM) has purportedly beneficial therapeutic effects on stage 5 CKD (nondialysis), which includes improving patients' clinical symptoms, slowing down disease progression, delaying the onset of dialysis and kidney transplantation, reducing certain complications, and improving quality of life. However, there is still a lack of high-quality evidence-based studies that support this, especially in mainland China. Therefore, in this study, through cooperation with multiple medical units across the country, a prospective nonrandomized controlled study was conducted to investigate the clinical value of CHM combined with Western medicine for slowing down disease progression and delaying the onset of dialysis in stage 5 CKD patients, and to provide an evidence-based foundation for the treatment of stage $5 \mathrm{CKD}$ with CHM.

We present the following article in accordance with the TREND reporting checklist (available at http://dx.doi. org/10.21037/atm-21-871).

\section{Methods}

\section{Study setting and patients}

This study was a multicenter prospective nonrandomized controlled study. From January 2013 to December 2017, patients with stage 5 CKD not undergoing dialysis were recruited from 29 AAA class hospitals (as shown in Table S1) across China. The study was conducted in accordance with the Declaration of Helsinki (as revised in 2013). All included patients provided written informed consent and the study protocol was approved by the Ethics Committee of Guangdong Provincial Hospital of Chinese Medicine (no. B2014-006-01; B2014-006-02). This study is registered at the Chinese Clinical Trial Registry (no. NCT02194946).

\section{Diagnostic criteria}

The diagnostic of CKD was based on the consensus of the Kidney Disease: Improving Global Outcomes (KDIGO) Clinical Practice Guideline (7). eGFR was estimated using the Chronic Kidney Disease Epidemiology Collaboration equation (CKD-EPI2009SCR) (8). The criteria for the clinical staging of CKD was as follows (7): stage 1: GFR $\geq 90 \mathrm{~mL} / \mathrm{min} / 1.73 \mathrm{~m}^{2}$; stage 2 : GFR $60-89.9 \mathrm{~mL} / \mathrm{min}$ $/ 1.73 \mathrm{~m}^{2}$; stage 3 : GFR $30-59.9 \mathrm{~mL} / \mathrm{min} / 1.73 \mathrm{~m}^{2}$; stage 4 : GFR $15-29.9 \mathrm{~mL} / \mathrm{min} / 1.73 \mathrm{~m}^{2}$; stage 5 : GFR $<15 \mathrm{~mL} / \mathrm{min}$ 
$/ 1.73 \mathrm{~m}^{2}$ (or dialysis).

\section{Inclusion and exclusion criteria}

The inclusion criteria were as follows: (I) patients aged 18-75 years old; (II) estimated (e)GFR $<15 \mathrm{~mL} / \mathrm{min}$ $/ 1.73 \mathrm{~m}^{2}$; (III) a primary disease of nondiabetic nephropathy; (IV) Asian ethnicity.

The exclusion criteria were as follows: (I) according to the Kidney Disease Outcome Quality Initiative (K-DOQI) guidelines, after conservative medical treatment, one of the following conditions still occurred: hemoglobin $(\mathrm{Hb})$ $<70 \mathrm{~g} / \mathrm{L}$, or carbon dioxide combining power $\left(\mathrm{CO}_{2} \mathrm{CP}\right)$ $<13 \mathrm{mmol} / \mathrm{L}$, or blood potassium $(\mathrm{K})>5.5 \mathrm{mmol} / \mathrm{L}$, or excessive levels of blood creatinine, or eGFR $\leq 5 \mathrm{~mL} / \mathrm{min}$ $/ 1.73 \mathrm{~m}^{2}$ (CKD-EPI formula); (II) pregnant or lactating patients; (III) patients with active malignant tumors, decompensated liver cirrhosis, hematopoietic diseases or other serious primary diseases, gastrointestinal bleeding, or psychotic patients; (IV) patients who had taken glucocorticoids or other immunosuppressants within the past 3 months; (V) patients who were participating in other clinical studies; (VI) patients who were known to be allergic or intolerant to a certain drug in this study; (VII) other reasons which researchers believed should exclude patients.

\section{Sample size estimation}

According to previous data from the Department of Nephrology of Guangdong Provincial Hospital of Chinese Medicine, the median time from recruitment to dialysis initiation was approximately 9.1 months in patients with CKD stage 5 in the CHM group. International research has shown that the time of late initiation of dialysis in patients treated with Western medicine to be delayed by approximately 6 months (5). An $\alpha=0.05, \beta=0.2$ in a bilateral test, and a loss to follow-up rate of $20 \%$ in each group with 3 years of treatment with $80 \%$ statistical power were considered necessary to prove that the time to dialysis onset for stage $5 \mathrm{CKD}$ patients in the CHM group is longer than that in the non-CHM group. Assuming a CHM to non$\mathrm{CHM}$ ratio of 3:1, we estimated that 881 participants would be required.

\section{Patient recruitment}

Patient recruitment was carried out by 2 researchers appointed by the heads of the research centers, and all recruited patients in this center were screened by the 2 appointed researchers. Patients were contacted in advance and asked to prepare recent (within 3 months) renal function test results and relevant medical records before recruitment. The recruiter recorded the patients' demographic information, renal function test results, and disease diagnosis when they first met with the patients. For eligible patients, if patients agreed, they would be immediately asked to sign a written informed consent form. Then, patients were divided into the CHM group (Western medicine + CHM) or non-CHM group (Western medicine) according to the doctors' recommendation and the patients' wishes.

\section{Control versus intervention}

The non-CHM group was treated with Western medicine, while the CHM group was treated with Western medicine + traditional Chinese medicine. The non-CHM group did not accept any Chinese medicine treatment, and the specific treatment plan was formulated in accordance with KDIGO and KDOQI guidelines, including nutritional therapy, blood pressure control, anemia treatment, regulating the metabolism of calcium and phosphorus, regulating the water, electrolyte and acid-base equilibrium, etc.

The traditional Chinese medicine treatment included oral Chinese medicine decoction, oral Chinese patent medicine, and traditional Chinese medicinal enemas, with all 3 being optional. These are described below.

(I) Oral Chinese medicine decoction: At each follow-up time point, researchers prescribed Chinese medicine for each patient after syndrome differentiation. The most common Chinese herbal medicine are Huang Qi (Radix astragali), Fu Ling (Poria), Bai Zhu (Rbizoma atractylodis macrocephalae), Shan Yao (Rhizoma dioscoreae), and Di Huang (Radix rehmanniae), ect.

(II) Oral Chinese patent medicine: Treatment was applied according to the patient's condition. There were generally no more than 3 types of oral Chinese patent medicine, and they were taken according to the drug instructions. The most common Chinese patent medicine includes Niaoduqing Particles, Sanqi oral liquid, Jinshuibao, Huangkui capsules, Bailing capsules, Shenshuaining, etc.

(III) Traditional Chinese medicinal enemas: Drugs were Chinese patent medicine, suppositories, or Chinese medicine decoctions used for the treatment 
of chronic renal failure or stage 5 CKD. The ingredients of the drugs were based on rhubarb, calcined oysters, and dandelion. The modes of intestinal administration were enema, colon dialysis, and colon administration. The specific steps were performed according to the drug instructions, and were taken $7-15$ times every 4 weeks.

After enrollment, patients should continue to take medication according to the study protocol until an endpoint event occurred or the end of the study was reached.

\section{Follow-up observation}

Patients were followed up every 4 weeks. General information was collected at the time of enrollment, including patient's serial number, abbreviated name, age, sex, height, weight, and other demographic data, as well as the primary disease. Data including blood pressure, and intake of Chinese and Western medicines, were recorded during monthly follow-up. Laboratory blood tests results were collected every 3 months, and were performed with a unified test method formulated by the Clinical Inspection Center of the Ministry of Health. The research team would regularly contact the patients by phone to reinforce the compliance with treatment and follow-up.

\section{Outcome assessment and safety evaluations}

The primary outcome of this study was the occurrence of dialysis or should initiate dialysis according to clinical judgment criteria. The first day of the outcome occurrence is set as the endpoint. Safety evaluations included assessments of adverse events/reactions and laboratory results. The researcher recorded all the adverse events/ reactions observed or reported by patients during the research process. Laboratory results included blood test results, routine stool examinations and occult blood, liver function, serum $\mathrm{K}, \mathrm{CO}_{2} \mathrm{CP}$, and electrocardiogram results. Adverse events and their severity were assessed by a professional nephrologists at each visit.

\section{Clinical judgment criteria of dialysis initiation}

Clinical judgment criteria of dialysis initiation included objective criteria and subjective criteria. The objective criteria included: (I) eGFR $\leq 5 \mathrm{~mL} / \mathrm{min} / 1.73 \mathrm{~m}^{2}$ (CKDEPI formula), (II) serum $\mathrm{K} \geq 6.5 \mathrm{mmol} / \mathrm{L}$, (III) metabolic acidosis, $\mathrm{CO}_{2} \mathrm{CP}<13 \mathrm{mmol} / \mathrm{L}$. The subjective criteria included: (I) uremic pericarditis or pleurisy, (II) uremic encephalopathy, (III) congestive heart failure or refractory hypertension, (IV) obvious bleeding tendency, (V) malnutrition that continues to deteriorate [including anorexia, weight loss, and decreased serum albumin (ALB)]. Clinically, if stage 5 CKD patients met the above criteria, we would recommend the patient to receive dialysis treatment, but if the patient did not receive dialysis due to financial or personal reasons, this study also thought that the patient had reached the endpoint.

\section{Data collection and data management}

After the research was completed and within 15 working days, each research unit submitted the finished case report forms (CRFs) into an electronic platform specially developed for this study, which was provided to the participating centers by the leading unit. Each research unit designated 2 data entry personnel, and all data were entered twice and double-checked to ensure consistency and accuracy. Finally, the research data was extracted from the electronic data platform and input into an Excel database.

\section{Statistical analysis}

Patient sex, age, primary disease, body mass index (BMI), pulse pressure difference, $\mathrm{ALB}, \mathrm{Hb}, \mathrm{K}, \mathrm{CO}_{2} \mathrm{CP}$, and eGFR were used as covariates in this study. Age, primary disease, $\mathrm{BMI}, \mathrm{ALB}, \mathrm{Hb}, \mathrm{K}, \mathrm{CO}_{2} \mathrm{CP}$, and eGFR were reclassified into categorical variables: 2 levels for the age factor $(<60$ and $\geq 60$ years), 4 levels for the primary disease factor (chronic nephritis syndrome, primary nephrotic syndrome, immunoglobin A nephropathy, and others), 3 levels for the BMI factor $\left(<18.5,18.5-23.9\right.$. and $\left.\geq 24 \mathrm{~kg} / \mathrm{m}^{2}\right), 2$ levels for the ALB factor $(\leq 35$ and $>35 \mathrm{~g} / \mathrm{L}), 2$ levels for the $\mathrm{Hb}$ factor $(\leq 115$ and $>115 \mathrm{~g} / \mathrm{L}), 3$ levels for the $\mathrm{K}$ factor $(\leq 3.5,3.5-5.5$, and $>5.5 \mathrm{mmol} / \mathrm{L}$ ), 2 levels for the $\mathrm{CO}_{2} \mathrm{CP}$ factor $(\leq 22$ and $>22 \mathrm{mmol} / \mathrm{L})$, and 2 levels for the eGFR factor $(<10$ and $\left.\geq 10 \mathrm{~mL} / \mathrm{min} / 1.73 \mathrm{~m}^{2}\right)$. For the baseline analysis, continuous variables conforming to a normal distribution are presented as mean \pm standard deviation, and were analyzed using a $t$-test. Continuous variables not conforming to the normal distribution are presented as median (interquartile range), and were compared using the two-sample Mann-Whitney $\mathrm{U}$ test. Categorical variables are presented as frequencies (percentage), and were compared using the chi-square test or Fisher's exact test. Univariate and multivariate Cox 
regression analyses were used to estimate the hazard ratios (HRs) and 95\% confidence interval (CI) of dialysis risk for CHM use. Because the number of cases of stage $5 \mathrm{CKD}$ non-CHM users was small, and the initial status of patients with stage 5 CKD might have been a confounding factor for the outcome, propensity score matching (PSM) was applied to eliminate baseline differences between traditional Chinese medicine (TCM) users and nonusers, including sex, age, primary disease, BMI, pulse pressure difference, $\mathrm{ALB}, \mathrm{Hb}$, serum $\mathrm{K}, \mathrm{CO}_{2} \mathrm{CP}$, and eGFR. All these viable covariates were used in the multiple logistic regression model to generate a score for each patient, and each eligible subject was matched according to the score by using the nearest-neighbor method at a 1:2 ratio with a caliper of 0.2 (9), and another matched cohort was acquired. For the matched cohort, baseline analysis was performed, and the standardized difference was calculated using the "stddiff" $\mathrm{R}$ package ( $\mathrm{R}$ Foundation for Statistical Computing, Vienna, Austria) before and after matching. Univariate and multivariate Cox regression analyses were performed, and a subgroup analysis was conducted that included factoring of sex, age, primary disease, $\mathrm{BMI}, \mathrm{ALB}, \mathrm{Hb}$, serum $\mathrm{K}, \mathrm{CO}_{2} \mathrm{CP}$, and eGFR to obtain HRs and 95\% CIs of dialysis risk in each CHM subgroup. The Kaplan-Meier method and log-rank tests were performed to compare the cumulative incidence rate of dialysis among the 2 groups before and after matching. Finally, we performed sensitivity tests with the PSM method using a caliper of 0.05 . Statistical analyses in this study were performed using the SPSS v.24.0 statistical software (IBM Corp., Armonk, NY, USA) and R v.3.6.2, with a significance level of $\mathrm{P}<0.05$.

\section{Results}

\section{Demographic characteristics}

From July 2014 to April 2019, a total of 908 patients with stage 5 CKD (nondialysis) were recruited from 29 AAA class hospitals from across China. Among these patients, 63 patients did not meet the inclusion criteria, with 25 patients lacking medication records and 6 patients undergoing dialysis within 4 weeks. Therefore, 814 patients were eligible for inclusion in the final analysis, of whom 747 were TCM users and 67 were nonusers (Figure 1). The median follow-up time was $6[3,12]$ months. The median age of patients was $51.00(41.00,62.00)$ years in the CHM group and $55.00(40.00,64.00)$ years in the non-CHM group. Male patients accounted for $49.4 \%$ and $64.2 \%$ in the CHM and non-CHM groups, respectively. The median baseline eGFR was $10.07(8.20-12.34) \mathrm{mL} / \mathrm{min} / 1.73 \mathrm{~m}^{2}$ in the CHM group and $8.70(6.84-10.21) \mathrm{mL} / \mathrm{min} / 1.73 \mathrm{~m}^{2}$ in the non-CHM group. Furthermore, the baseline values of sex, primary disease, BMI, pulse pressure difference, $\mathrm{K}$, and eGFR were significantly different between the 2 groups (Table 1).

\section{Survival analysis}

The survival analysis results showed a total of 534 dialysis events occurred among all study participants, including 52 non-CHM users and $482 \mathrm{CHM}$ users. The median time of dialysis initiation in the non-CHM group was $3(0.98,5.02)$ months, and the median time of dialysis initiation in the CHM group was $9(7.90,10.10)$ months. The Kaplan-Meier analysis demonstrated that the cumulative incidence of dialysis in the CHM group was significantly lower than that in the non-CHM group (log-rank test, $\mathrm{P}<0.001$; Figure 2).

\section{Multivariate Cox regression analysis}

The multivariate Cox regression analysis showed that the incidence rate of dialysis was significantly lower in stage 5 CKD patients using CHM than that in stage $5 \mathrm{CKD}$ patients not using CHM (aHR: 0.38; 95\% CI: 0.28, 0.53; $\mathrm{P}<0.001$; Table 2).

\section{Propensity score matching}

The results of the baseline analysis (Table 1) showed that there were significant differences regarding the baseline characteristics between the 2 groups. Therefore, PSM was applied to eliminate the baseline differences between TCM users and nonusers. After 1:2 matching, the final study population consisted of $106 \mathrm{CHM}$ users and 54 non-CHM users. The median age of patients was 55.50 (46.00-66.00) years in the CHM group and $52.00(40.00-63.00)$ years in the non-CHM group. Male patients accounted for $57.5 \%$ and $63.0 \%$ of the CHM and non-CHM groups, respectively. The median baseline eGFR was 8.73 (7.78$10.30) \mathrm{mL} / \mathrm{min} / 1.73 \mathrm{~m}^{2}$ in the CHM group and 8.93 $(6.30-10.50) \mathrm{mL} / \mathrm{min} / 1.73 \mathrm{~m}^{2}$ in the non-CHM group. The baseline demographic characteristics of TCM users were quite similar to those of non-users. Except for ALB, the standardized differences after matching were all improved compared to before matching, and the standardized differences of age, pulse pressure, $\mathrm{Hb}, \mathrm{CO}_{2} \mathrm{CP}$, and eGFR 


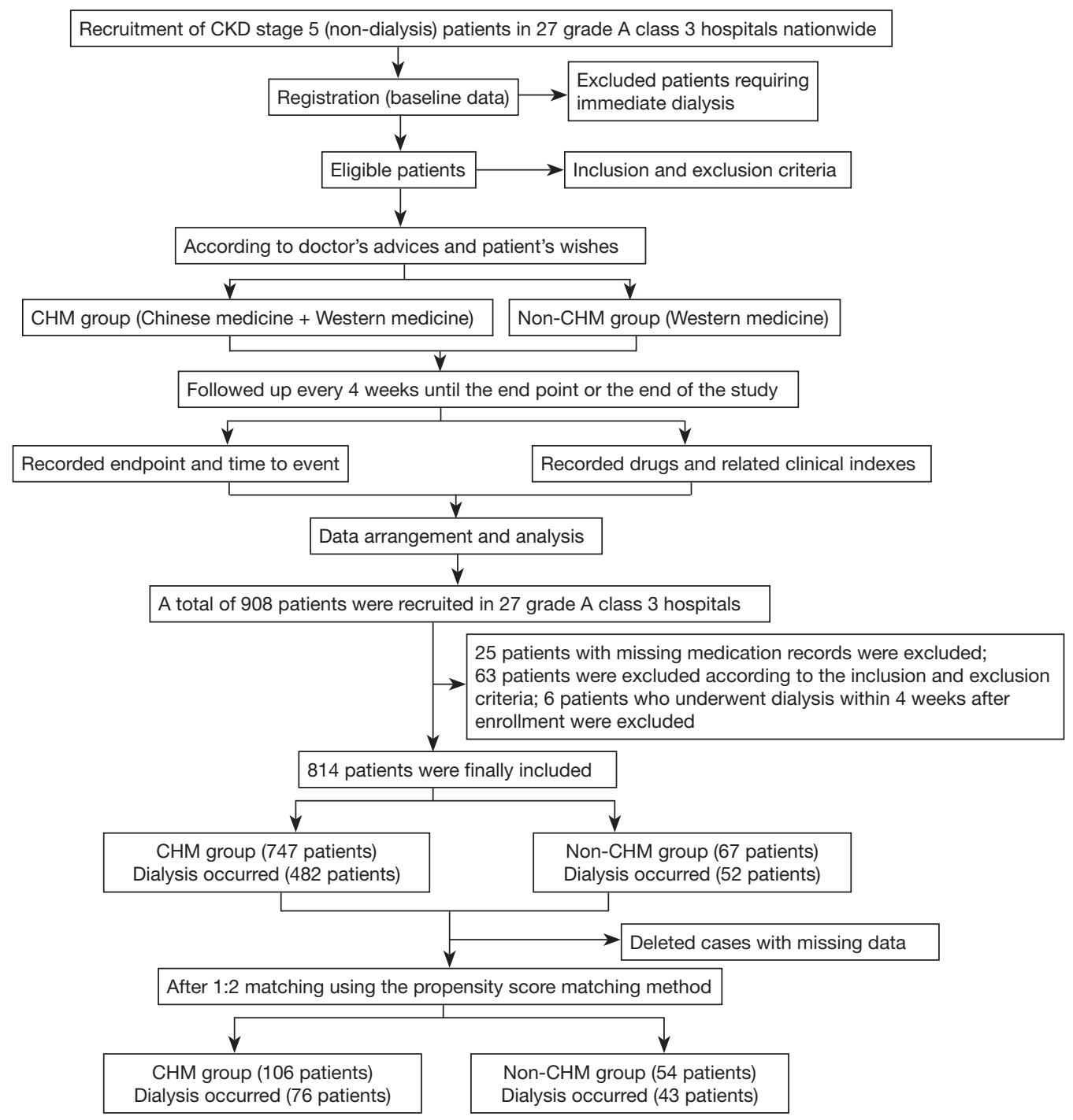

Figure 1 Flow chart of this study. CKD, chronic kidney disease; CHM, Chinese herbal medicine.

after matching were all lower than 10\% (Table 3).

\section{Survival analysis after 1:2 matching}

The survival analysis results showed that a total of 119 dialysis events occurred among all study participants, including 43 non-CHM users and 76 CHM users. The median time of initiating dialysis in the non-CHM group was $3(1.14,4.86)$ months, and the median time of initiating dialysis in the CHM group was $9(7.43,10.57)$ months. The Kaplan-Meier analysis demonstrated that the cumulative incidence of dialysis in the CHM group was significantly lower than that in the non-CHM group (log-rank test,
$\mathrm{P}<0.001$; Figure 3).

\section{Hazard ratios and cumulative incidence rates of dialysis for the CHM and non-CHM coborts after 1:2 matching}

The multivariate Cox regression analysis showed that the incidence rate of dialysis was significantly lower in the stage 5 CKD patients using CHMs than in the stage 5 CKD patients not using CHMs (aHR: 0.32; 95\% CI: 0.21, 0.48; $\mathrm{P}<0.001)$. Patients with $\mathrm{ALB}>35 \mathrm{~g} / \mathrm{L}$ were at a significantly lower risk for developing dialysis than those with ALB $\leq 35$ g/L (aHR: 0.42; 95\% CI: 0.23, 0.76; P<0.01). Patients with $\mathrm{Hb}>115 \mathrm{~g} / \mathrm{L}$ were at a significantly lower risk for 
Table 1 Baseline characteristics of the enrolled patients with stage 5 CKD in the 2 groups

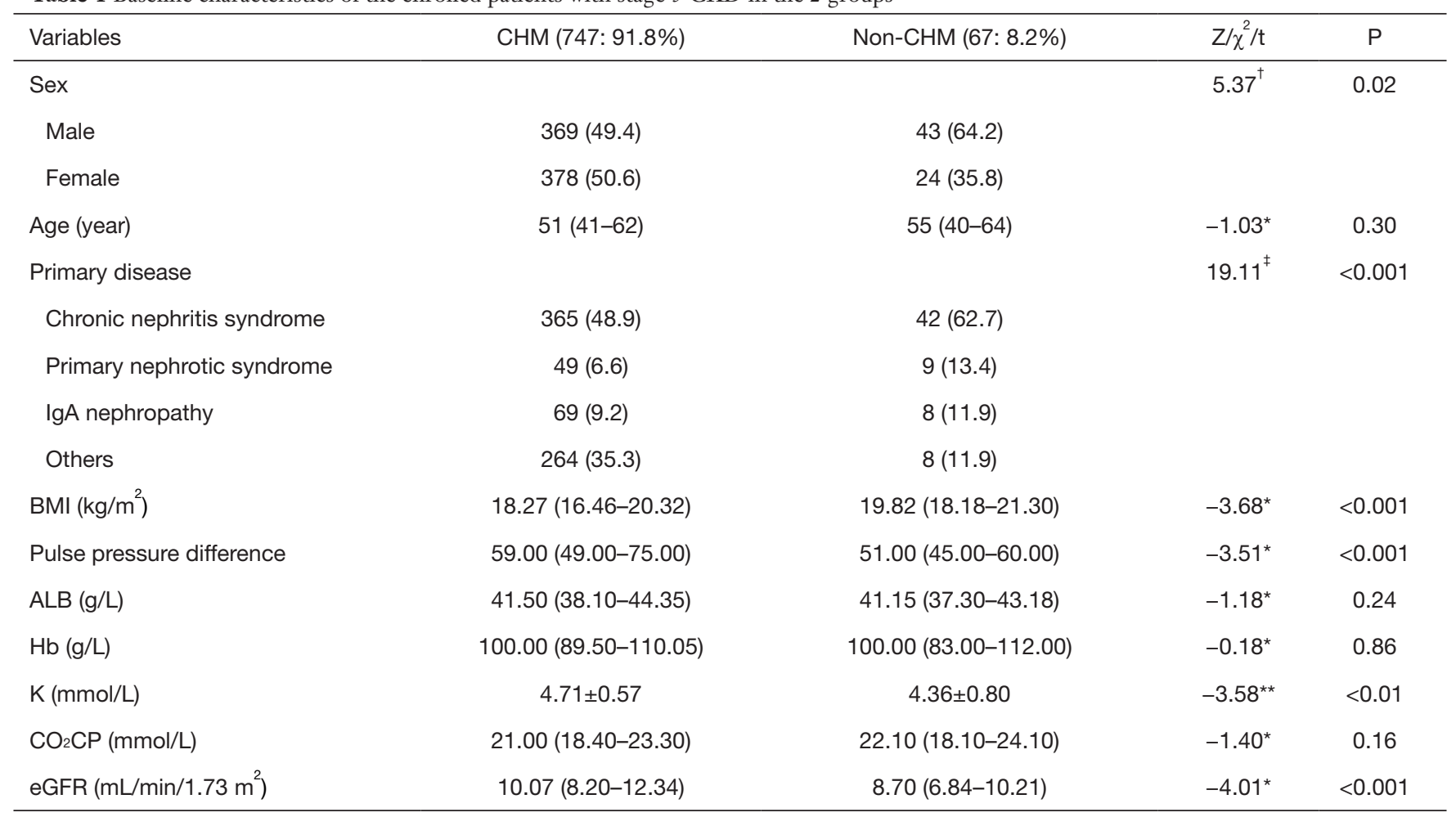

Values are given as $\mathrm{n}(\%)$, mean \pm standard deviation, or median (P25-P75). ${ }^{*}$, Mann-Whitney $U$ test; ${ }^{* *}, t$-tests; ${ }^{\dagger}$, Chisquare test; ${ }^{*}$, Fisher's exact tests. CKD. chronic kidney disease; CHM, Chinese herbal medicine; BMI, body mass index; ALB, albumin; Hb, hemoglobin; $\mathrm{K}$, serum potassium; $\mathrm{CO}_{2} \mathrm{CP}$, carbon dioxide combining power; eGFR, estimated glomerular filtration rate.

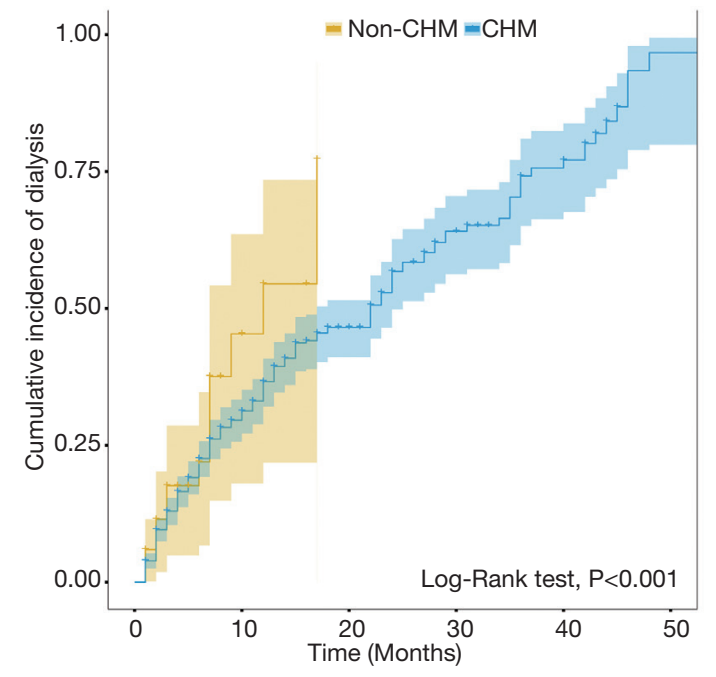

Figure 2 Cumulative incidence rate of dialysis among patients with stage $5 \mathrm{CKD}$ in the CHM and non-CHM groups. CKD, chronic kidney disease; CHM, Chinese herbal medicine. developing dialysis than those with $\mathrm{Hb} \leq 115 \mathrm{~g} / \mathrm{L}$ (aHR: 0.63; 95\% CI: 0.39, 0.99; $\mathrm{P}<0.05)$. Furthermore, patients with $\mathrm{CO}_{2} \mathrm{CP}>22 \mathrm{mmol} / \mathrm{L}$ were at a significantly lower risk for developing dialysis than those with $\mathrm{CO}_{2} \mathrm{CP} \leq 22 \mathrm{mmol} / \mathrm{L}$ (aHR: 0.56; 95\% CI: 0.38, 0.82; $\mathrm{P}<0.01$ ). Patients with eGFR $\geq 10 \mathrm{~mL} / \mathrm{min} / 1.73 \mathrm{~m}^{2}$ were at a significantly lower risk for developing dialysis than those with eGFR $<10 \mathrm{~mL} / \mathrm{min}$ $/ 1.73 \mathrm{~m}^{2}$ (aHR: 0.46; 95\% CI: 0.30, 0.73; P<0.01; Table 4).

\section{Subgroup analysis of the risk of dialysis}

The subgroup analysis showed that CHM users had a lower risk of dialysis compared to non-users among stratifications of sex, age, $\mathrm{ALB}, \mathrm{CO}_{2} \mathrm{CP}$, and eGFR. In addition, in the subgroups of chronic nephritis syndrome, $\mathrm{Hb} \leq 115 \mathrm{~g} / \mathrm{L}$, $3.5 \mathrm{mmol} / \mathrm{L}<\mathrm{K} \leq 5.5 \mathrm{mmol} / \mathrm{L}, \mathrm{BMI}<18.5 \mathrm{~kg} / \mathrm{m}^{2}$, and $18.5 \mathrm{~kg} / \mathrm{m}^{2} \leq \mathrm{BMI}<23.9 \mathrm{~kg} / \mathrm{m}^{2}$, patients in the CHM group 
Table 2 Cox model measured hazard ratio and $95 \%$ confidence intervals of dialysis associated with and without CHM and covariates among stage 5 CKD patients

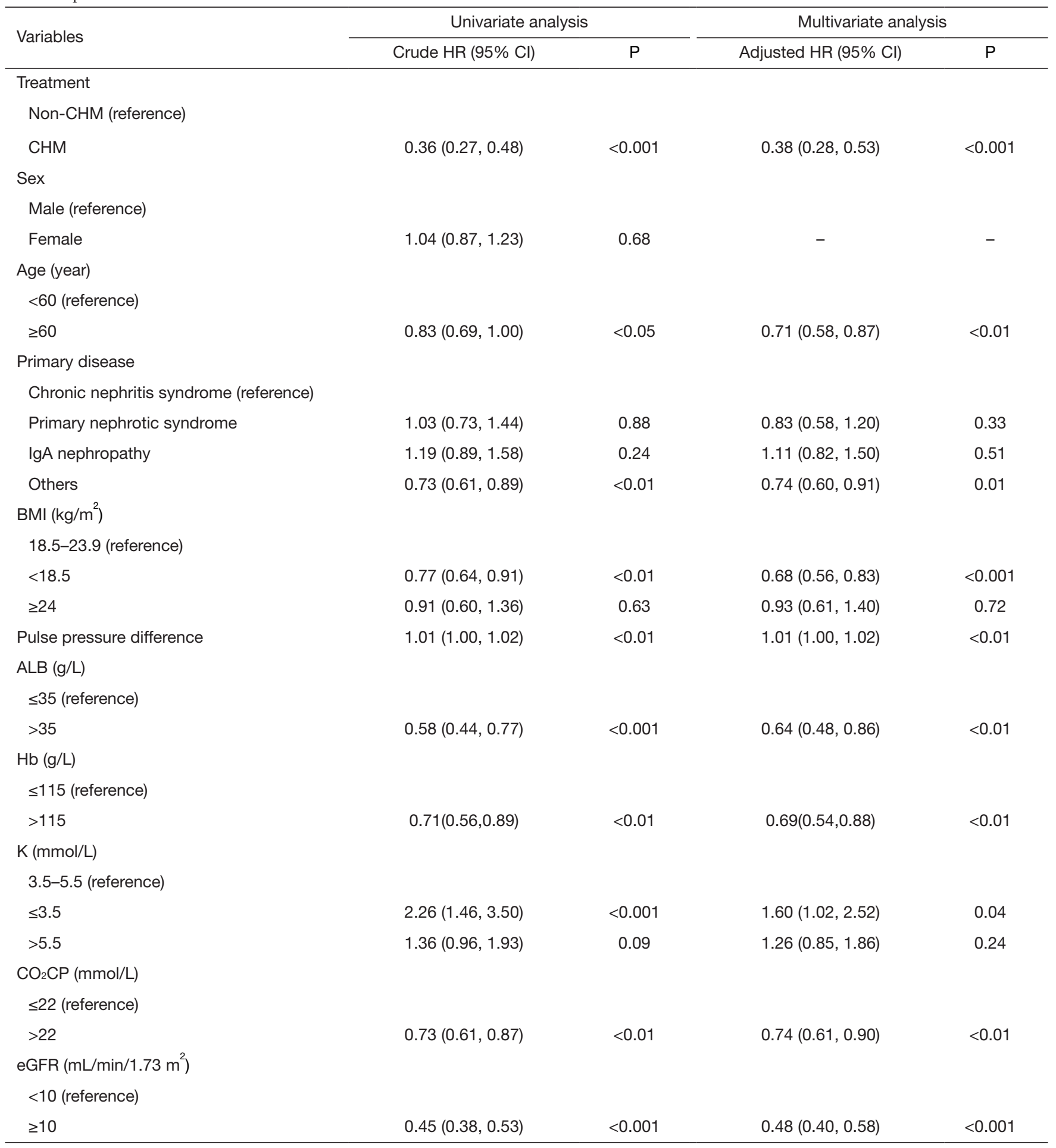

Crude HR: represented relative hazard ratio; adjusted HR(aHR): represented adjusted hazard ratio, adjusted for $\mathrm{CHM}$ use, age, primary disease, $\mathrm{BMI}$, pulse pressure, $\mathrm{ALB}, \mathrm{Hb}, \mathrm{K}, \mathrm{CO}_{2} \mathrm{CP}$, and eGFR in the multivariate Cox regression analysis. CKD, chronic kidney disease; $\mathrm{HR}$, hazard ratio; $\mathrm{Cl}$, confidence interval; $\mathrm{CHM}$, Chinese herbal medicine; BMI, body mass index; ALB, albumin; Hb, hemoglobin; K, serum potassium; $\mathrm{CO}_{2} \mathrm{CP}$, carbon dioxide combining power; eGFR, estimated glomerular filtration rate. 
Table 3 Baseline characteristics of the enrolled patients with stage 5 CKD in the 2 groups (after 1:2 matching)

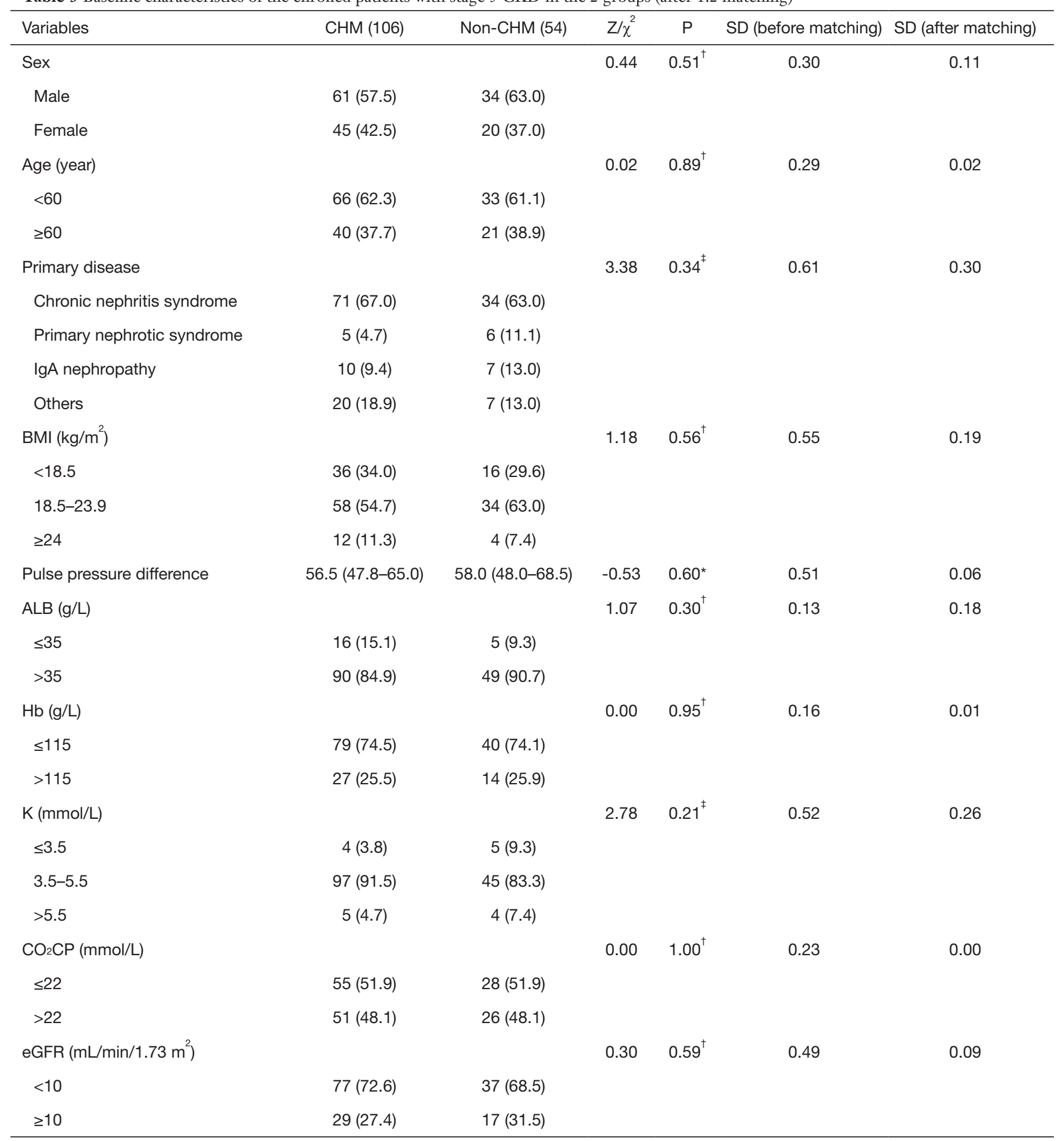

Values are given as $\mathrm{n}(\%)$, or median (P25-P75). ${ }^{*}$, Mann-Whitney $\mathrm{U}$ test; ${ }^{\dagger}$, Chisquare test; ${ }^{\ddagger}$, Fisher's exact test. CKD, chronic kidney disease; $\mathrm{CHM}$, Chinese herbal medicine; $\mathrm{BMI}$, body mass index; $\mathrm{ALB}$, albumin; $\mathrm{Hb}$, hemoglobin; $\mathrm{K}$, serum potassium; $\mathrm{CO}_{2} \mathrm{CP} \mathrm{Carbon}$ dioxide combining power; eGFR, estimated glomerular filtration rate; SD, standardized difference. 


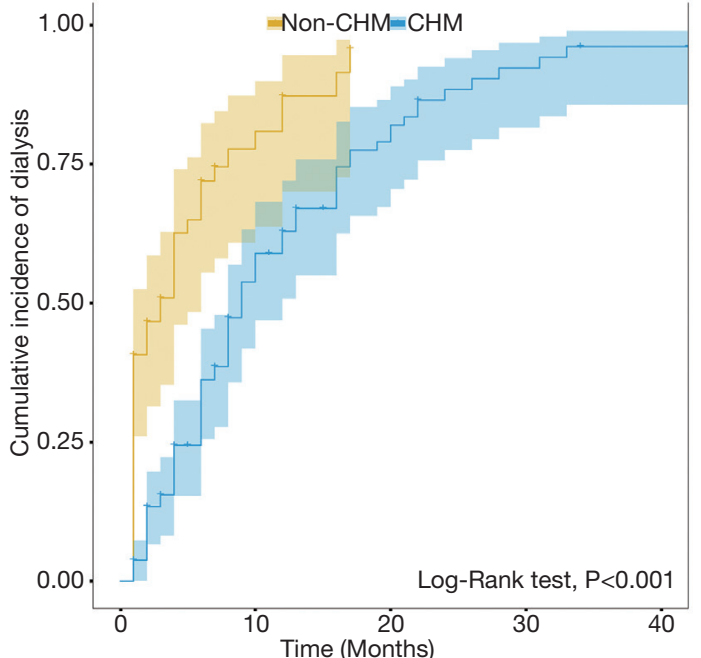

Figure 3 Cumulative incidence rate of dialysis among patients with stage $5 \mathrm{CKD}$ in the CHM and non-CHM groups (after 1:2 matching). CKD, chronic kidney disease; CHM, Chinese herbal medicine.

had a significantly lower risk of dialysis compared to those in the non-CHM group (Figure 4).

\section{Sensitivity tests}

The sensitivity tests firmly supported the results. The results were similar in the 2 cohorts matched by PSM with different calipers. When the caliper was 0.05 , the multivariate Cox regression analysis showed that the incidence rate of dialysis was significantly lower in stage $5 \mathrm{CKD}$ patients using CHM than in stage $5 \mathrm{CKD}$ patients not using CHM (aHR: 0.47; 95 \% CI: 0.30, 0.72; $\mathrm{P}<0.01)$. The Kaplan-Meier analysis demonstrated that the cumulative incidence of dialysis in the CHM group was significantly lower than that in the non-CHM group (logrank test, $\mathrm{P}<0.001$; Table S2, Table S3, Figure $\mathrm{S} 1$ ).

\section{Safety}

Adverse events occurred in 233 patients in the CHM group and 4 patients in the non-CHM group. The main adverse events, which included hyperkalemia (160 patients in the CHM group, 2 patients in the non-CHM group), upper respiratory tract infection (24 patients in the CHM group, 0 patients in the non-CHM group), and metabolic acidosis (9 patients in the CHM group, 0 patients in the non-CHM group), all showed no significant differences between the
2 groups $(\mathrm{P}>0.05)$. Other adverse events, listed in Table $\mathrm{S} 4$, also showed no significant differences between both groups ( $>>0.05$; Table S4).

\section{Discussion}

In China, traditional Chinese medicine (TCM) has been widely used in medical practice for thousands of years, and has been remarkably effective for treating diseases using various methods. It is also a core part of Chinese culture, and is well accepted by Chinese people. Therefore, patients who treat their diseases with TCM constitute a large proportion in China, including CKD patients. According to published research (10), $45 \%$ of CKD patients are treated with TCM in Taiwan. Due to this social phenomenon, although there were 12 Western hospitals among the 29 AAA class hospitals in our study, the overall use rate of TCM still reached $92 \%$, which indicated a high use rate of TCM in Western hospitals in China.

In clinical practice, studies have repeatedly confirmed the beneficial effects of TCM as a treatment for CKD. It has been shown to help patients by relieving several clinical symptoms, including pruritus, fatigue, depression, anxiety, uremic, and bruising, preventing cardiovascular complications, and even delaying CKD progression (11); however, there is a lack of large-scale high-quality evidencebased medical evidence for these benefits. The published studies have all thus far been from Taiwan, China. A study with data collected from 2000 to 2008 showed that patients with CKD taking aristolochic acid-free TCM herbs had a lower risk of mortality (aHR: 0.6, 95\% CI: 0.5, 0.7) compared to nonusers (12). During a similar study period, research by Lin et al. (10) showed that, compared with nonuser group, the end-stage renal disease (ESRD) risk significantly reduced by $60 \%$ in the user group adjusted [cause-specific hazard ratio (aCSHR): $0.41,95 \%$ CI: 0.37 , 0.46]. In addition, research by Huang et al. (13) indicated that patients with CKD using CHM treatments exhibited an increased long-term survival rate within a 12 -year follow-up period $(\mathrm{P}<0.004)$. Meanwhile, a study by Chen et al. (14) showed that TCM users might have a lower mortality and ESRD rate among patients with incident diabetic nephropathy.

However, there are few multicenter, high-quality, largesample, published clinical studies regarding the efficacy of TCM treatment for CKD in inland China. Therefore, this study focused on patients with stage $5 \mathrm{CKD}$, and aimed to observe the effects of Chinese medicine combined with 
Table 4 Cox model measured hazard ratio and $95 \%$ confidence intervals of dialysis associated with and without CHM and covariates among stage 5 CKD patients (after 1:2 matching)

\begin{tabular}{|c|c|c|c|c|}
\hline \multirow{2}{*}{ Variables } & \multicolumn{2}{|c|}{ Univariate analysis } & \multicolumn{2}{|c|}{ Multivariate analysis } \\
\hline & Crude HR $(95 \% \mathrm{Cl})$ & $P$ & Adjusted HR (95\% Cl) & $\mathrm{P}$ \\
\hline \multicolumn{5}{|l|}{ Treatment } \\
\hline $\mathrm{CHM}$ & $0.41(0.27,0.60)$ & $<0.001$ & $0.32(0.21,0.48)$ & $<0.001$ \\
\hline \multicolumn{5}{|l|}{ Sex } \\
\hline Female & $1.14(0.79,1.65)$ & 0.48 & - & - \\
\hline \multicolumn{5}{|l|}{ Age (year) } \\
\hline \multicolumn{5}{|l|}{$<60$ (reference) } \\
\hline$\geq 60$ & $0.79(0.54,1.14)$ & 0.21 & - & - \\
\hline IgA nephropathy & $1.42(0.80,2.53)$ & 0.23 & - & - \\
\hline Others & $0.71(0.42,1.18)$ & 0.19 & - & - \\
\hline \multicolumn{5}{|l|}{ BMI $\left(\mathrm{kg} / \mathrm{m}^{2}\right)$} \\
\hline \multicolumn{5}{|l|}{ 18.5-23.9 (reference) } \\
\hline$<18.5$ & $0.74(0.50,1.09)$ & 0.13 & - & - \\
\hline$\geq 24$ & $0.53(0.26,1.05)$ & 0.07 & - & - \\
\hline Pulse pressure difference & $1.01(1.00,1.02)$ & 0.16 & - & - \\
\hline \multicolumn{5}{|l|}{ ALB (g/L) } \\
\hline \multicolumn{5}{|l|}{$\mathrm{K}(\mathrm{mmol} / \mathrm{L})$} \\
\hline \multicolumn{5}{|l|}{ 3.5-5.5 (reference) } \\
\hline$\leq 3.5$ & $1.81(0.84,3.91)$ & 0.13 & - & - \\
\hline$>5.5$ & $1.29(0.56,2.94)$ & 0.55 & - & - \\
\hline \multicolumn{5}{|l|}{$\mathrm{CO}_{2} \mathrm{CP}(\mathrm{mmol} / \mathrm{L})$} \\
\hline \multicolumn{5}{|l|}{$\leq 22$ (reference) } \\
\hline$>22$ & $0.53(0.37,0.78)$ & $<0.01$ & $0.56(0.38,0.82)$ & $<0.01$ \\
\hline \multicolumn{5}{|l|}{ eGFR $\left(\mathrm{mL} / \mathrm{min} / 1.73 \mathrm{~m}^{2}\right)$} \\
\hline \multicolumn{5}{|l|}{$<10$ (reference) } \\
\hline$\geq 10$ & $0.47(0.30,0.73)$ & $<0.01$ & $0.46(0.30,0.73)$ & $<0.01$ \\
\hline
\end{tabular}

Crude HR: represented relative hazard ratio; adjusted HR: represented adjusted hazard ratio, adjusted for $\mathrm{CHM}_{\mathrm{H} e}, \mathrm{ALB}, \mathrm{Hb}, \mathrm{CO}_{2} \mathrm{CP}$, and eGFR in the multivariate Cox regression analysis. CKD, chronic kidney disease; HR, hazard ratio; Cl, confidence interval; $\mathrm{CHM}$, Chinese herbal medicine; BMI, body mass index; ALB, albumin; $\mathrm{Hb}$, hemoglobin; $\mathrm{K}$, serum potassium; $\mathrm{CO}_{2} \mathrm{CP}$, carbon dioxide combining power; eGFR, estimated glomerular filtration rate. 


\begin{tabular}{|c|c|c|c|c|c|c|c|}
\hline Variables & CHM & Events Non & -CHM & Events & & $\mathrm{HR}(95 \% \mathrm{CI})$ & $P$ \\
\hline \multicolumn{8}{|l|}{ Sex } \\
\hline Male & 61 & 42 & 34 & $\rightarrow$ & & $0.37(0.22,0.63)$ & $<0.001$ \\
\hline Female & 45 & 34 & 20 & 17 & & $0.47(0.25,0.86)$ & 0.01 \\
\hline \multicolumn{8}{|l|}{ Age (year) } \\
\hline$<60$ & 66 & 46 & 33 & $\rightarrow$ & & $0.37(0.22,0.61)$ & $<0.001$ \\
\hline$\geq 60$ & 40 & 30 & 21 & $\rightarrow$ & & $0.42(0.22,0.81)$ & 0.01 \\
\hline \multicolumn{8}{|l|}{ Primary Disease } \\
\hline Chronic nephritis syndrome & 71 & 52 & 34 & $\rightarrow$ & & $0.43(0.26,0.69)$ & $<0.001$ \\
\hline Primary nephrotic syndrome & 5 & 2 & 6 & 5 & & $0.16(0.02,1.43)$ & 0.10 \\
\hline IgA nephropathy & 10 & 8 & 7 & 6 & & $0.52(0.17,1.61)$ & 0.26 \\
\hline Others & 20 & 14 & 7 & 4 & & $0.55(0.17,1.76)$ & 0.31 \\
\hline \multicolumn{8}{|l|}{ BMI $\left(\mathrm{kg} / \mathrm{m}^{2}\right)$} \\
\hline$<18.5$ & 36 & 26 & 16 & 13 & & $0.42(0.21,0.85)$ & 0.02 \\
\hline $18.5-23.9$ & 58 & 43 & 34 & 28 & & $0.43(0.26,0.71)$ & $<0.01$ \\
\hline$\geq 24$ & 12 & 7 & 4 & 2 & & $0.14(0.01,1.52)$ & 0.11 \\
\hline \multicolumn{8}{|l|}{$\operatorname{ALB}(g / L)$} \\
\hline$\leq 35$ & 16 & 9 & 5 & 5 & & $0.22(0.06,0.78)$ & 0.02 \\
\hline$>35$ & 90 & 67 & 49 & $\rightarrow$ & & $0.40(0.26,0.61)$ & $<0.001$ \\
\hline \multicolumn{8}{|l|}{$\mathrm{Hb}(\mathrm{g} / \mathrm{L})$} \\
\hline$\leq 115$ & 79 & 58 & 40 & $\rightarrow$ & & $0.37(0.24,0.59)$ & $<0.001$ \\
\hline$>115$ & 27 & 18 & 14 & $\longrightarrow$ & 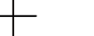 & $0.53(0.23,1.22)$ & 0.14 \\
\hline \multicolumn{8}{|l|}{$\mathrm{K}(\mathrm{mmol} / \mathrm{L})$} \\
\hline$\leq 3.5$ & 4 & 3 & 5 & 4 & & $0.20(0.02,1.89)$ & 0.16 \\
\hline $3.5-5.5$ & 97 & 71 & 45 & $\rightarrow$ & & $0.45(0.29,0.68)$ & $<0.001$ \\
\hline$>5.5$ & 5 & 2 & 4 & 4 & & $0.12(0.01,1.12)$ & 0.06 \\
\hline \multicolumn{8}{|l|}{$\mathrm{CO}_{2} \mathrm{CP}(\mathrm{mmol} / \mathrm{L})$} \\
\hline$\leq 22$ & 55 & 42 & 28 & 27 & & $0.25(0.15,0.43)$ & $<0.001$ \\
\hline$>22$ & 51 & 34 & 26 & 16 & & $0.51(0.27,0.96)$ & 0.04 \\
\hline \multicolumn{8}{|l|}{ eGFR (mL/min/1.73 m²) } \\
\hline$<10$ & 77 & 60 & 37 & $\rightarrow$ & & $0.39(0.25,0.62)$ & $<0.001$ \\
\hline \multirow[t]{2}{*}{$\geq 10$} & 29 & 16 & 17 & 10 & & $0.30(0.12,0.72)$ & 0.01 \\
\hline & & & & 0.5 & 1.5 & 2 & \\
\hline
\end{tabular}

Figure 4 Subgroup analysis of the impact of CHM use on dialysis occurrence. — - - represents hazard ratio and $95 \%$ confidence interval. CHM, Chinese herbal medicine; BMI, body mass index; $\mathrm{ALB}$, albumin; Hb, hemoglobin; $\mathrm{K}$, serum potassium; $\mathrm{CO}_{2} \mathrm{CP}_{\text {, carbon dioxide }}$ combining power; eGFR, estimated glomerular filtration rate. Variables were not adjusted in the Cox regression model of subgroup analysis.

Western medicine on the onset of dialysis events, and whether it helped to delay disease progression. Our research showed that, compared with Western medicine, treatment with Chinese medicine combined with Western medicine resulted in a significantly lower risk of dialysis before and after matching, and the median time to dialysis in the CHM group was about 6 months later than that in the non-CHM group. The Kaplan-Meier analysis also demonstrated that the cumulative incidence of dialysis in the CHM group was significantly lower than that in the non-CHM group before and after matching. Results of this study and the above studies in Taiwan all indicate favorable clinical outcomes of TCM treatment for CKD.Chronic renal failure is a kind of clinical syndrome characterized by kidney dysfunction. Traditional Chinese medicine considered that its main cause may be spleen-kidney Qi deficiency, which could result in a kind of pathological condition of the obstruction of the metabolism of Qi, blood and body fluid and the process of energy transformation, and retention of dampness, phlegm and blood stasis in the body. Traditional Chinese medicine could effectively relieve this pathological condition and improve renal function by strengthening spleen and tonifying kidney, and removing dampness, phlegm and blood stasis.

He et al. collected prescriptions for the treatment of renal injury in CHM clinical reports from databases such as the China National Knowledge Infrastructure (CNKI), VIP, and Wanfang. Frequency statistics were performed on herbs among all the prescriptions recorded, and the association rules analysis showed that the core herbs included Huang Qi (Radix astragali), Fu Ling (Poria), Bai Zhu (Rbizoma atractylodis macrocephalae), Shan Yao (Rhizoma dioscoreae), and Di Huang (Radix rehmanniae) (15), which was generally consistent with the clinical medications of 
this study. Huang Qi, Fu Ling, Bai Zhu, and Shan Yao strengthen the spleen, benefit Qi, and remove dampness. Di Huang nourishes Yin and supplements kidneys. At the same time, modern pharmacological studies have shown that Astragaloside IV, derived from Radix astragali, could protect against kidney injury by ameliorating albuminuria, attenuating podocyte apoptosis, and inhibiting oxidative stress and nuclear factor kappa B

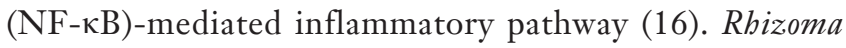
atractylodis macrocephalae was found to have a diuretic effect by inhibiting water reabsorption (17), attenuating muscle wasting in CKD via the oxidative stress-mediated pathway (18), and improving glomerular lesions (19). Novel renin-angiotensin system (RAS) inhibitors isolated from Poria could inhibit renin and were found to protect against tubulointerstitial fibrosis (20), while lanostane triterpenoids from the sclerotia of Poria cocos Wolf were shown to have protective effect against cell death in LLC-PK1 kidney tubular epithelial cells (21). Furthermore, Poria also has a diuretic effect, which can be used for treating chronic edema and kidney disease (22). Dioscin extracted from Dioscoreae rhizoma has been shown to improve fructoseinduced renal injury by up-regulating Sirt3 signal to inhibit inflammation, oxidative stress, lipid metabolism, and renal fibrosis (23). Another study (24) also found that dioscin could significantly inhibit the proliferation of THP-1cells, obviously reduce the soakage of inflammatory cells and necrosis in the kidney of rats and mice, and significantly decline the levels of creatinine ( $\mathrm{Cr}$ ), blood urea nitrogen (BUN). Catalpol is the chief active component extracted from the root of Radix rebmanniae, Research found that Catalpol could protect podocytes from injury by inhibiting NOX4 to improve apoptosis and inflammatory response (25). Research also discovered that, acteoside, the main component of Radix rehmanniae, could alleviate mesangial cell inflammatory injury by modulating Th22 lymphocytes chemotaxis and proliferation (26).

Furthermore, results of the multivariate Cox regression analysis in this study showed that patients with ALB $>35 \mathrm{~g} / \mathrm{L}$ were at a significantly lower risk than those with ALB $\leq 35 \mathrm{~g} / \mathrm{L}$ for developing dialysis. ALB is one of the biomarkers of protein wasting energy (PEW). A large number of studies have confirmed that low ALB is significantly related to the death of CKD (nondialysis) patients and the occurrence of ESRD (27-29). A study by Amdur et al. (30) indicated that reduced ALB was significantly associated with reduced eGFR (HR: 1.52, 95\% CI: $1.24,1.87)$. Meanwhile, in Tangri et al.'s (31) prediction model research, ALB was found to be significant predictor for CKD patients initiating renal replacement therapy (RRT) treatment. Thus, all these findings suggest that low ALB is an important risk factor for poor prognosis in CKD.

Our results also indicated that patients with $\mathrm{Hb}$ $>115 \mathrm{~g} / \mathrm{L}$ had a significantly lower risk for developing dialysis than those with $\mathrm{Hb} \leq 115 \mathrm{~g} / \mathrm{L}$. Research has shown that renal anemia is a crucial risk factor leading to the progression of nephropathy, the occurrence of cardiovascular events, and mortality events $(32,33)$, while other studies have found that correcting anemia can significantly slow down disease progression and delay the time to dialysis initiation $(34,35)$. Hence, patients with normal $\mathrm{Hb}$ levels have a lower risk of dialysis, which is consistent with our study results.

Patients with $\mathrm{CO}_{2} \mathrm{CP}>22 \mathrm{mmol} / \mathrm{L}$ were at a significantly lower risk for developing dialysis than those with $\mathrm{CO}_{2} \mathrm{CP}$ $\leq 22 \mathrm{mmol} / \mathrm{L}$. Studies have found that metabolic acidosis was related to the progression of CKD and the occurrence of death events (36-38). Dobre et al. (39) found that patients with serum bicarbonate $<22 \mathrm{mmol} / \mathrm{L}$ had an almost a 2 -fold increased risk of renal disease progression (HR: 1.97; 95\% CI: $1.50,2.57)$. A study by Kovesdy et al. (40) revealed that, among non-dialysis CKD patients, when serum bicarbonate concentration $<22 \mathrm{mEq} / \mathrm{L}$, the mortality rate was the highest. Therefore, $\mathrm{CO}_{2} \mathrm{CP} \leq 22 \mathrm{mmol} / \mathrm{L}$ is also an important risk factor for the poor prognosis of CKD.

Patients with eGFR $\geq 10 \mathrm{~mL} / \mathrm{min} / 1.73 \mathrm{~m}^{2}$ were at significantly lower risk for developing dialysis than those with eGFR $<10 \mathrm{~mL} / \mathrm{min} / 1.73 \mathrm{~m}^{2}$, which is in line with clinical practice. When eGFR $<10 \mathrm{~mL} / \mathrm{min}$ $/ 1.73 \mathrm{~m}^{2}$, serious symptoms and signs of uremic toxin accumulation and volume overload begin to appear, and dialysis is immediately required (41). Research by Lin et al. (42) also revealed that a lower baseline eGFR was an independent and significant risk factor for reaching dialysis treatment in CKD stages 3b-5 patients.

The subgroup analysis showed that CHM users had a lower risk of dialysis compared to nonusers among stratifications of age, sex, ALB, $\mathrm{CO}_{2} \mathrm{CP}$, and eGFR. After stratification by primary disease, patients with chronic nephritis syndrome in the CHM group had a significantly lower risk of dialysis compared to those in the non-CHM group. However, no significant difference was observed in this respect among patients with primary nephrotic syndrome or immunoglobin A (IgA) nephropathy or other causes between the CHM and non-CHM groups. This may be because the primary disease of most patients in this study 
was chronic nephritis syndrome (accounting for $50 \%$ ), and after 1:2 matching, the statistical sample size of primary disease with nephrotic syndrome, IgA nephropathy, and other causes were smaller, which resulted in lower statistical power and potential false-negative results.

After stratification by BMI, patients with BMI $<18.5 \mathrm{~kg} / \mathrm{m}^{2}$ or $18.5 \mathrm{~kg} / \mathrm{m}^{2} \leq \mathrm{BMI}<23.9 \mathrm{~kg} / \mathrm{m}^{2}$ in the CHM group had a significantly lower risk of dialysis compared to their counterparts in the non-CHM group. However, no significant difference was observed for patients with BMI $\geq 24 \mathrm{~kg} / \mathrm{m}^{2}$ between the CHM and non-CHM groups (43). A previous study demonstrated that higher BMI was not a risk factor for predicting renal replacement therapy in patients with CKD. A "reverse epidemiology" phenomenon (44-46), has been proposed, in which obesity in predialysis and hemodialysis populations may protect against all-cause mortality. We therefore speculate that higher BMI may not be an important risk factor of dialysis for CKD patients. Thus, when $B M I \geq 24 \mathrm{~kg} / \mathrm{m}^{2}$, no significant difference was seen between the 2 groups.

After stratification by $\mathrm{Hb}$, patients with $\mathrm{Hb} \leq 115 \mathrm{~g} / \mathrm{L}$ in the CHM group had a significantly lower risk of dialysis compared to those in the non-CHM group, while no significant difference was observed in this respect among patients with $\mathrm{Hb}>115 \mathrm{~g} / \mathrm{L}$ between the CHM and nonCHM groups. Studies have demonstrated that certain Chinese medicines have the effect of replenishing Qi and blood, which can effectively improve anemia (47), while the correction of anemia is beneficial for delaying disease progression and slowing the need for maintenance dialysis $(34,35)$. Therefore, when $\mathrm{Hb} \leq 115 \mathrm{~g} / \mathrm{L}$, our study observed that the risk of dialysis was significantly reduced after CHM treatment.

After stratification by $\mathrm{K}$, patients with $3.5 \mathrm{mmol} / \mathrm{L}$ $<\mathrm{K} \leq 5.5 \mathrm{mmol} / \mathrm{L}$ in the CHM group had a significantly lower risk of dialysis compared to those in the non-CHM group. However, no significant difference was observed in this respect among patients with $\mathrm{K} \leq 3.5 \mathrm{mmol} / \mathrm{L}$ and $\mathrm{K}$ $>5.5 \mathrm{mmol} / \mathrm{L}$ between the CHM and non-CHM groups. This may be because the statistical sample size of $\mathrm{K}$ $\leq 3.5 \mathrm{mmol} / \mathrm{L}$ and $\mathrm{K}>5.5 \mathrm{mmol} / \mathrm{L}$ were too smaller to reach the statistical differences.

However, overall, the combination of CHM and Western medicine could effectively reduce the incidence of dialysis in patients with stage $5 \mathrm{CKD}$ and prolong the time of dialysis initiation. Hence, we suggest that in actual clinical practice, when stage 5 CKD patients see a doctor, doctors with TCM qualifications can prescribe TCM treatment to each patient following the principles of syndrome differentiation and treatment, after a thorough examination of the patient's symptoms and signs and after their consent.

The participants in this study were recruited from across China, providing a good representation of the Chinese population. Therefore, the conclusions of this study are applicable to Chinese patients with stage 5 CKD. However, stage 5 CKD patients in this study did not include patients with diabetic nephropathy, because a previous study suggested that patients with diabetic nephropathy progressed rapidly (42). In patients with diabetic nephropathy, the rate of GFR decline generally exceeds $10 \mathrm{~mL} / \mathrm{min}$ per year, and even reaches $10-20 \mathrm{~mL} / \mathrm{min}$ per year $(48-52)$. Therefore, the time to dialysis initiation will be significantly shorter for patients with diabetic nephropathy compared to nondiabetic nephropathy patients. Furthermore, in order to evaluate the effect of TCM on the time to dialysis initiation for patients with stage 5 CKD more objectively, this study excluded patients with diabetic nephropathy.

There are some shortcomings in this study. Firstly, the data collected at the end of this study showed that the proportion of CHM users and non-CHM users was significantly unbalanced. Although PSM analysis was used, this imbalance greatly reduces the sample size for statistical analysis and statistical power. Secondly, all patients selected in this study were Chinese. Therefore, further studies are needed to identify the clinical benefits of TCM treatment in other populations. Thirdly, this study did not further analyze the characteristics and prescription patterns of CHM use in patients with CKD, which will be investigated in our subsequent research. In the future, we will further explore safe, effective, and economical TCM treatments for nondialysis stage 5 CKD patients, which are suitable for clinical promotion and popularization. Fourthly, the exact mechanisms of the active components of the Chinese medicine used in this study still require further research. Additionally, detailed information such as patients' lifestyles were not collected in this study, so we could not clarify whether CHM-users tended to maintain healthier lifestyles, which could influence disease progression and the time of dialysis initiation in CKD patients. Lastly, due to the short follow-up period, this study did not observe and record the effects of TCM treatment on other endpoint events such as cardiovascular events, stroke, and death in stage $5 \mathrm{CKD}$ patients. We will continue to follow up with the patients in our center who participated in this study and evaluate other possible clinical benefits of TCM treatment on patients 
with advanced renal disease.

\section{Conclusions}

This study was a multicenter, prospective, nonrandomized controlled study based on stage 5 CKD patients that aimed to evaluate the effects of Chinese medicine combined with Western medicine on the time to dialysis initiation and the occurrence of dialysis events as compared to Western medicine. The results of this study suggest that Chinese medicine combined with Western medicine can effectively reduce the incidence of dialysis among stage 5 CKD patients, slow kidney disease progression, and extend the time to dialysis initiation.

\section{Acknowledgments}

Funding: This work was supported and funded by the Ministry of Science and Technology of the People's Republic of China (no. 2013BAI02B04). State Key Laboratory of Dampness Syndrome of Chinese Medicine, The Second Affiliated Hospital of Guangzhou University of Chinese Medicine (no. SZ2020ZZ22), and Special Project of Guangdong Provincial Key Laboratory of Clinical Research on Traditional Chinese Medicine Syndrome (no. ZH2020KF02).

\section{Footnote}

Reporting Checklist: The authors have completed the TREND reporting checklist. Available at http://dx.doi. org/10.21037/atm-21-871

Data Sharing Statement: Available at http://dx.doi. org/10.21037/atm-21-871

Conflicts of Interest: All authors have completed the ICMJE uniform disclosure form (available at http://dx.doi. org/10.21037/atm-21-871). The authors have no conflicts of interest to declare.

Ethical Statement: The authors are accountable for all aspects of the work in ensuring that questions related to the accuracy or integrity of any part of the work are appropriately investigated and resolved. The study was conducted in accordance with the Declaration of Helsinki (as revised in 2013). All included patients provided written informed consent and the study protocol was approved by the Ethics Committee of Guangdong Provincial Hospital of Chinese Medicine (no. B2014-006-01; B2014-006-02). This study is registered at the Chinese Clinical Trial Registry (no. NCT02194946).

Open Access Statement: This is an Open Access article distributed in accordance with the Creative Commons Attribution-NonCommercial-NoDerivs 4.0 International License (CC BY-NC-ND 4.0), which permits the noncommercial replication and distribution of the article with the strict proviso that no changes or edits are made and the original work is properly cited (including links to both the formal publication through the relevant DOI and the license). See: https://creativecommons.org/licenses/by-nc-nd/4.0/.

\section{References}

1. Hill NR, Fatoba ST, Oke JL, et al. Global Prevalence of Chronic Kidney Disease - A Systematic Review and MetaAnalysis. PLoS One 2016;11:e158765.

2. Bikbov B, Purcell CA, Levey AS, et al. Global, regional, and national burden of chronic kidney disease, 1990-2017: a systematic analysis for the Global Burden of Disease Study 2017. Lancet 2020;395:709-33.

3. KDIGO KDIGOKCWG. 2012 clinical practice guideline for the evaluation and management of chronic kidney disease. Kidney Int Suppl 2013;3:1-150.

4. Tattersall J, Dekker F, Heimburger O, et al. When to start dialysis: updated guidance following publication of the Initiating Dialysis Early and Late (IDEAL) study. Nephrol Dial Transplant 2011;26:2082-6.

5. Cooper BA, Branley P, Bulfone L, et al. A Randomized, Controlled Trial of Early versus Late Initiation of Dialysis. N Engl J Med 2010;363:609-19.

6. Rosansky SJ, Eggers P, Jackson K, et al. Early start of hemodialysis may be harmful. Arch Intern Med 2011;171:396-403.

7. Inker LA, Astor BC, Fox CH, et al. KDOQI US commentary on the 2012 KDIGO clinical practice guideline for the evaluation and management of CKD. Am J Kidney Dis 2014;63:713-35.

8. Levey AS, Stevens LA, Schmid CH, et al. A new equation to estimate glomerular filtration rate. Ann Intern Med 2009;150:604-12.

9. Austin PC. Optimal caliper widths for propensity-score matching when estimating differences in means and differences in proportions in observational studies. Pharm Stat 2011;10:150-61. 
10. Lin MY, Chiu YW, Chang JS, et al. Association of prescribed Chinese herbal medicine use with risk of endstage renal disease in patients with chronic kidney disease. Kidney Int 2015;88:1365-73.

11. Markell MS. Potential benefits of complementary medicine modalities in patients with chronic kidney disease. Adv Chronic Kidney Dis 2005;12:292-9.

12. Hsieh CF, Huang SL, Chen CL, et al. Non-aristolochic acid prescribed Chinese herbal medicines and the risk of mortality in patients with chronic kidney disease: results from a population-based follow-up study. BMJ Open 2014;4:e004033.

13. Huang KC, Su YC, Sun MF, et al. Chinese Herbal Medicine Improves the Long-Term Survival Rate of Patients With Chronic Kidney Disease in Taiwan: A Nationwide Retrospective Population-Based Cohort Study. Front Pharmacol 2018;9:1117.

14. Chen HY, Pan HC, Chen YC, et al. Traditional Chinese medicine use is associated with lower end-stage renal disease and mortality rates among patients with diabetic nephropathy: a population-based cohort study. BMC Complement Altern Med 2019;19:81.

15. He T, Qu RD, Qin CM, et al. Potential mechanisms of Chinese Herbal Medicine that implicated in the treatment of COVID-19 related renal injury. Saudi Pharm J 2020;28:1138-48.

16. Zhang J, Wu C, Gao L, et al. Astragaloside IV derived from Astragalus membranaceus: A research review on the pharmacological effects. Adv Pharmacol 2020;87:89-112.

17. Lee YP, Lee YJ, Lee SM, et al. Effect of Atractylodes macrocephala on Hypertonic Stress-Induced Water Channel Protein Expression in Renal Collecting Duct Cells. Evid Based Complement Alternat Med 2012;2012:650809.

18. Wang M, Hu R, Wang Y, et al. Atractylenolide III Attenuates Muscle Wasting in Chronic Kidney Disease via the Oxidative Stress-Mediated PI3K/AKT/mTOR Pathway. Oxid Med Cell Longev 2019;2019:1875471.

19. Ishii T, Okuyama T, Noguchi N, et al. Antiinflammatory constituents of Atractylodes chinensis rhizome improve glomerular lesions in immunoglobulin A nephropathy model mice. J Nat Med 2020;74:51-64.

20. Wang M, Chen DQ, Chen L, et al. Novel inhibitors of the cellular renin-angiotensin system components, poricoic acids, target Smad3 phosphorylation and Wnt/betacatenin pathway against renal fibrosis. Br J Pharmacol 2018;175:2689-708.

21. Lee D, Lee S, Shim SH, et al. Protective effect of lanostane triterpenoids from the sclerotia of Poria cocos Wolf against cisplatin-induced apoptosis in LLC-PK1 cells. Bioorg Med Chem Lett 2017;27:2881-5.

22. Lee SM, Lee YJ, Yoon JJ, et al. Effect of Poria cocos on hypertonic stress-induced water channel expression and apoptosis in renal collecting duct cells. J Ethnopharmacol 2012;141:368-76.

23. Qiao Y, Xu L, Tao X, et al. Protective effects of dioscin against fructose-induced renal damage via adjusting Sirt3mediated oxidative stress, fibrosis, lipid metabolism and inflammation. Toxicol Lett 2018;284:37-45.

24. Zhao X, Yin L, Fang L, et al. Protective effects of dioscin against systemic inflammatory response syndromevia adjusting TLR2/MyD88/NFkappab signal pathway. Int Immunopharmacol 2018;65:458-69.

25. Chen Y, Liu Q, Shan Z, et al. The protective effect and mechanism of catalpol on high glucose-induced podocyte injury. BMC Complement Altern Med 2019;19:244.

26. Gan L, Li XZ, Zhu MY, et al. Acteoside relieves mesangial cell injury by regulating Th22 cell chemotaxis and proliferation in IgA nephropathy. Renal Failure 2018;40:364-70.

27. Menon V, Greene T, Wang XL, et al. C-reactive protein and albumin as predictors of all-cause and cardiovascular mortality in chronic kidney disease. Kidney Int 2005;68:766-72.

28. Kovesdy CP, George SM, Anderson JE, et al. Outcome predictability of biomarkers of protein-energy wasting and inflammation in moderate and advanced chronic kidney disease. Am J Clin Nutr 2009;90:407-14.

29. Weiner DE, Tighiouart H, Elsayed EF, et al. The relationship between nontraditional risk factors and outcomes in individuals with stage 3 to 4 CKD. Am J Kidney Dis 2008;51:212-23.

30. Amdur RL, Feldman HI, Gupta J, et al. Inflammation and Progression of CKD: The CRIC Study. Clin J Am Soc Nephrol 2016;11:1546-56.

31. Tangri N, Inker LA, Hiebert B, et al. A Dynamic Predictive Model for Progression of CKD. Am J Kidney Dis 2017;69:514-20.

32. He J, Shlipak M, Anderson A, et al. Risk Factors for Heart Failure in Patients With Chronic Kidney Disease: The CRIC (Chronic Renal Insufficiency Cohort) Study. J Am Heart Assoc 2017;6:e005336.

33. Stirnadel-Farrant HA, Luo J, Kler L, et al. Anemia and mortality in patients with nondialysis-dependent chronic kidney disease. BMC Nephrol 2018;19:135.

34. Jungers P, Choukroun G, Oualim Z, et al. Beneficial 
influence of recombinant human erythropoietin therapy on the rate of progression of chronic renal failure in predialysis patients. Nephrol Dial Transplant 2001;16:307-12.

35. Kuriyama S, Tomonari H, Yoshida H, et al. Reversal of anemia by erythropoietin therapy retards the progression of chronic renal failure, especially in nondiabetic patients. Nephron 1997;77:176-85.

36. Menon V, Tighiouart H, Vaughn NS, et al. Serum Bicarbonate and Long-term Outcomes in CKD. Am J Kidney Dis 2010;56:907-14.

37. Kraut JA, Madias NE. Metabolic Acidosis of CKD: An Update. Am J Kidney Dis 2016;67:307-17.

38. Shah SN, Abramowitz M, Hostetter TH, et al. Serum Bicarbonate Levels and the Progression of Kidney Disease: A Cohort Study. Am J Kidney Dis 2009;54:270-7.

39. Dobre M, Yang W, Pan Q, et al. Persistent high serum bicarbonate and the risk of heart failure in patients with chronic kidney disease (CKD): A report from the Chronic Renal Insufficiency Cohort (CRIC) study. J Am Heart Assoc 2015;4:e001599.

40. Kovesdy CP, Anderson JE, Kalantar-Zadeh K. Association of serum bicarbonate levels with mortality in patients with non-dialysis-dependent CKD. Nephrol Dial Transplant 2009;24:1232-7.

41. Chen T, Lee VW, Harris DC. When to initiate dialysis for end-stage kidney disease: evidence and challenges. Med J Aust 2018;209:275-9.

42. Lin CM, Yang MC, Hwang SJ, Sung JM. Progression of stages 3b-5 chronic kidney disease--preliminary results of Taiwan national pre-ESRD disease management program in Southern Taiwan. J Formos Med Assoc 2013;112:773-82.

43. Wang Z, Zhang J, Chan S, et al. BMI and its association with death and the initiation of renal replacement therapy (RRT) in a cohort of patients with chronic kidney disease (CKD). BMC Nephrol 2019;20:329.

44. Beddhu S. The body mass index paradox and an obesity, inflammation, and atherosclerosis syndrome in chronic kidney disease. Semin Dial 2004;17:229-32.

45. Ladhani $M$, Craig JC, Irving $M$, et al. Obesity and the risk of cardiovascular and all-cause mortality in chronic kidney disease: a systematic review and meta-analysis. Nephrol
Dial Transplant 2017;32:439-49.

46. Navaneethan SD, Schold JD, Arrigain S, et al. Body mass index and causes of death in chronic kidney disease. Kidney Int 2016;89:675-82.

47. Dang Z, Liu X, Wang X, et al. Comparative effectiveness and safety of traditional Chinese medicine supporting Qi and enriching blood for cancer related anemia in patients not receiving chemoradiotherapy: a meta-analysis and systematic review. Drug Des Devel Ther 2018;13:221-30.

48. Parving HH, Smidt UM, Hommel E, et al. Effective antihypertensive treatment postpones renal insufficiency in diabetic nephropathy. Am J Kidney Dis 1993;22:188-95.

49. Mogensen CE. Progression of nephropathy in longterm diabetics with proteinuria and effect of initial anti-hypertensive treatment. Scand J Clin Lab Invest 1976;36:383-8.

50. Parving HH, Smidt UM, Friisberg B, et al. A prospective study of glomerular filtration rate and arterial blood pressure in insulin-dependent diabetics with diabetic nephropathy. Diabetologia 1981;20:457-61.

51. Viberti GC, Bilous RW, Mackintosh D, et al. Monitoring glomerular function in diabetic nephropathy. A prospective study. Am J Med 1983;74:256-64.

52. Rossing P, Hommel E, Smidt UM, et al. Impact of arterial blood pressure and albuminuria on the progression of diabetic nephropathy in IDDM patients. Diabetes 1993;42:715-9.

Cite this article as: $\mathrm{Wu} \mathrm{Y,} \mathrm{Li} \mathrm{C,} \mathrm{Zhang} \mathrm{L,} \mathrm{Zou} \mathrm{C,} \mathrm{Xu} \mathrm{P,}$ Wen Z, Ouyang W, Yang N, Zhang M, Lin Q, Lu F, Wang L, Bao K, Zhao D, Fu L, Guo X, Yang L, Ou A, He Z, Weng H, Li J, Shi W, Wang X, Song L, Zhan Y, Sun W, Wei L, Wang N, Gui D, Zhan J, Lu Y, Chen H, Liu Y, Yang H, Chen M, Wang Y, Zhang P, Deng Y, Meng L, Cheng X, Li F, Yu D, Xu D, Fang J, Li H, Fu J, Xie Y, Li W, Zhao J, Huang Y, Lu Z, Su G, Zhang L, Qin X, Xu Y, Peng Y, Hou H, Deng L, Liu H, Jie X, Liu L, Tang F, Pei H, Li P, Mao W, Liu X. Effectiveness of Chinese herbal medicine combined with Western medicine on deferring dialysis initiation for nondialysis chronic kidney disease stage 5 patients: a multicenter prospective nonrandomized controlled study. Ann Transl Med 2021;9(6):490. doi: 10.21037/atm-21-871 
Table S1 The list of 29 AAA class hospitals participating in this study

\begin{tabular}{|c|c|}
\hline Number & Hospital Name \\
\hline 1 & Guangdong Provincial Hospital of Chinese Medicine \\
\hline 2 & First Affiliated Hospital of Guangxi University of Chinese Medicine \\
\hline 3 & Hubei Provincial Hospital of Chinese Medicine \\
\hline 4 & First Affiliated Hospital of Heilongjiang University Of Chinese Medicine \\
\hline 5 & Guang'anmen Hospital China Academy of Traditional Chinese Medicine \\
\hline 6 & Jiangsu Provincial Hospital of Chinese Medicine \\
\hline 7 & TCM Integrated Hospital of Southern Medical University \\
\hline 8 & The Sixth People's Hospital Affiliated to Shanghai Jiao Tong University \\
\hline 9 & First Affiliated Hospital of Guiyang College of Traditional Chinese Medicine \\
\hline 10 & Tong De Hospital, Zhejiang Province \\
\hline 11 & Hangzhou Hospital of Chinese Medicine \\
\hline 12 & Dongzhimen Hospital of Beijing University of Chinese Medicine \\
\hline 13 & Affiliated Hospital of Chengdu University of Traditional Chinese Medicine \\
\hline 14 & First Affiliated Hospital of Tianjin University Of Chinese Medicine \\
\hline 15 & Anhui Provincial Hospital of Chinese Medicine \\
\hline 16 & Heilongjiang Academy of Traditional Chinese Medicine \\
\hline 17 & Longhua Hospital Affiliated to Shanghai University of Traditional Chinese Medicine \\
\hline 18 & Liuzhou Hospital of traditional Chinese Medicine \\
\hline 19 & Shaanxi Provincial Hospital of Chinese Medicine \\
\hline 20 & Xijing Hospital of The Fourth Military Medical University \\
\hline 21 & Xiyuan Hospital, Academy of Traditional Chinese Medicine \\
\hline 22 & First Hospital of Peking University \\
\hline 23 & First hospital of Shanxi Medical University \\
\hline 24 & Huadu District People's Hospital of Guangzhou \\
\hline 25 & Guangzhou No.1 People's Hospital \\
\hline 26 & China PLA General Hospital \\
\hline 27 & China-Japan Friendship Hospital \\
\hline 28 & General Hospital of Guangzhou Military Command of PLA \\
\hline 29 & Third Military Medical University Xinqiao Hospital \\
\hline
\end{tabular}


Table S2 Baseline characteristics among patients in the CHM group and non-CHM group (after 1:2 matching with a caliper of 0.05)

\begin{tabular}{|c|c|c|c|c|c|}
\hline Variables & $\mathrm{CHM}(104)$ & Non-CHM (55) & $\mathrm{Z} / \mathrm{x}^{2}$ & $\mathrm{P}$ & SD (after matching) \\
\hline Sex & & & 0.00 & $0.98^{\dagger}$ & 0.00 \\
\hline Male & $66(63.5)$ & $35(63.6)$ & & & \\
\hline Female & $38(36.5)$ & $20(36.4)$ & & & \\
\hline Age (year) & & & 0.09 & $0.77^{\dagger}$ & 0.05 \\
\hline$<60$ & $58(55.8)$ & 32 (58.2) & & & \\
\hline$\geq 60$ & $46(44.2)$ & $23(41.8)$ & & & \\
\hline Primary disease & & & 8.83 & $0.03^{\ddagger}$ & 0.49 \\
\hline Chronic nephritis syndrome & $75(72.1)$ & $33(60.0)$ & & & \\
\hline Primary nephrotic syndrome & $7(6.7)$ & $8(14.5)$ & & & \\
\hline IgA nephropathy & $3(2.9)$ & $7(12.7)$ & & & \\
\hline Others & 19 (18.3) & $7(12.7)$ & & & \\
\hline $\mathrm{BMI}\left(\mathrm{kg} / \mathrm{m}^{2}\right)$ & & & 1.68 & $0.44^{\ddagger}$ & 0.22 \\
\hline$<18.5$ & $40(38.5)$ & $16(29.1)$ & & & \\
\hline $18.5-23.9$ & $55(52.9)$ & $35(63.6)$ & & & \\
\hline$\geq 24$ & $9(8.7)$ & $4(7.3)$ & & & \\
\hline Pulse pressure difference & $53.0(47.3-63.8)$ & $58.0(48.0-70.0)$ & -0.98 & $0.33^{\star}$ & 0.16 \\
\hline ALB (g/L) & & & 0.23 & $0.64^{\dagger}$ & 0.08 \\
\hline$\leq 35$ & $12(11.5)$ & $5(9.1)$ & & & \\
\hline$>35$ & $92(88.5)$ & $50(90.9)$ & & & \\
\hline $\mathrm{Hb}(\mathrm{g} / \mathrm{L})$ & & & 0.04 & $0.85^{\dagger}$ & 0.03 \\
\hline$\leq 115$ & $78(75.0)$ & $42(76.4)$ & & & \\
\hline$>115$ & $26(25.0)$ & $13(23.6)$ & & & \\
\hline $\mathrm{K}(\mathrm{mmol} / \mathrm{L})$ & & & 12.41 & $0.00^{\ddagger}$ & 0.55 \\
\hline$\leq 3.5$ & $1(1.0)$ & $8(14.5)$ & & & \\
\hline $3.5-5.5$ & $98(94.2)$ & $43(78.2)$ & & & \\
\hline$>5.5$ & $5(4.8)$ & $4(7.3)$ & & & \\
\hline $\mathrm{CO}_{2} \mathrm{CP}(\mathrm{mmol} / \mathrm{L})$ & & & 0.01 & $0.92^{\dagger}$ & 0.02 \\
\hline$\leq 22$ & $54(51.9)$ & $29(52.7)$ & & & \\
\hline$>22$ & $50(48.1)$ & $26(47.3)$ & & & \\
\hline $\operatorname{eGFR}\left(\mathrm{mL} / \mathrm{min} / 1.73 \mathrm{~m}^{2}\right)$ & & & 0.02 & $0.89^{\dagger}$ & 0.02 \\
\hline$<10$ & 73 (70.2) & $38(69.1)$ & & & \\
\hline$\geq 10$ & $31(29.8)$ & 17 (30.9) & & & \\
\hline
\end{tabular}

Values are given as $\mathrm{n}(\%)$, or median (P25-P75). ${ }^{*}$, Mann-Whitney $\mathrm{U}$ test; ${ }^{\dagger}$, chisquare test; ${ }^{\ddagger}$, Fisher's exact test. CHM, Chinese herbal medicine; $\mathrm{BMI}$, body mass index; ALB, albumin; $\mathrm{Hb}$, hemoglobin; $\mathrm{K}$, serum potassium; $\mathrm{CO}_{2} \mathrm{CP}$, carbon dioxide combining power; eGFR, estimated glomerular filtration rate; SD, standardized difference. 
Table S3 Cox model measured hazard ratio and 95\% confidence intervals of dialysis associated with and without CHM and covariates among stage 5 CKD patients (after 1:2 matching with a caliper of 0.05 )

\begin{tabular}{|c|c|c|c|c|}
\hline \multirow{2}{*}{ Variables } & \multicolumn{2}{|c|}{ Univariate analysis } & \multicolumn{2}{|c|}{ Multivariate analysis } \\
\hline & Crude HR (95\% Cl) & $\mathrm{P}$ & Adjusted HR (95\% Cl) & $\mathrm{P}$ \\
\hline \multicolumn{5}{|l|}{ Treatment } \\
\hline \multicolumn{5}{|l|}{ Non-CHM (reference) } \\
\hline $\mathrm{CHM}$ & $0.46(0.31,0.68)$ & $<0.001$ & $0.47(0.30,0.72)$ & $<0.01$ \\
\hline \multicolumn{5}{|l|}{ Sex } \\
\hline \multicolumn{5}{|l|}{ Male (reference) } \\
\hline Female & $1.14(0.78,1.66)$ & 0.49 & - & - \\
\hline \multicolumn{5}{|l|}{ Age (year) } \\
\hline \multicolumn{5}{|l|}{$<60$ (reference) } \\
\hline$\geq 60$ & $0.73(0.50,1.07)$ & 0.10 & - & - \\
\hline \multicolumn{5}{|l|}{ Primary disease } \\
\hline \multicolumn{5}{|c|}{ Chronic nephritis syndrome (reference) } \\
\hline Primary nephrotic syndrome & $2.09(1.09,3.99)$ & 0.03 & $1.77(0.92,3.41)$ & 0.09 \\
\hline IgA nephropathy & $1.27(0.61,2.64)$ & 0.52 & $1.27(0.59,2.74)$ & 0.54 \\
\hline Others & $0.64(0.37,1.12)$ & 0.12 & $0.65(0.37,1.15)$ & 0.14 \\
\hline \multicolumn{5}{|l|}{ BMI $\left(k g / m^{2}\right)$} \\
\hline \multicolumn{5}{|l|}{ 18.5-23.9 (reference) } \\
\hline$<18.5$ & $0.78(0.53,1.16)$ & 0.22 & - & - \\
\hline$\geq 24$ & $0.71(0.32,1.55)$ & 0.39 & - & - \\
\hline Pulse pressure difference & $1.01(1.00,1.02)$ & 0.18 & - & - \\
\hline \multicolumn{5}{|l|}{ ALB (g/L) } \\
\hline \multicolumn{5}{|l|}{$\leq 35$ (reference) } \\
\hline$>35$ & $0.55(0.28,1.07)$ & 0.08 & - & - \\
\hline \multicolumn{5}{|l|}{$\mathrm{Hb}(\mathrm{g} / \mathrm{L})$} \\
\hline \multicolumn{5}{|l|}{$\leq 115$ (reference) } \\
\hline$>115$ & $0.34(0.21,0.57)$ & $<0.001$ & $0.44(0.26,0.74)$ & $<0.01$ \\
\hline \multicolumn{5}{|l|}{$\mathrm{K}(\mathrm{mmol} / \mathrm{L})$} \\
\hline \multicolumn{5}{|l|}{ 3.5-5.5 (reference) } \\
\hline$\leq 3.5$ & $3.90(1.73,8.77)$ & $<0.01$ & $1.97(0.84,4.59)$ & 0.12 \\
\hline$>5.5$ & $2.73(1.17,6.41)$ & 0.02 & $1.40(0.58,3.38)$ & 0.45 \\
\hline \multicolumn{5}{|l|}{$\mathrm{CO}_{2} \mathrm{CP}(\mathrm{mmol} / \mathrm{L})$} \\
\hline \multicolumn{5}{|l|}{$\leq 22$ (reference) } \\
\hline$>22$ & $0.43(0.29,0.64)$ & $<0.001$ & $0.57(0.37,0.86)$ & 0.01 \\
\hline \multicolumn{5}{|l|}{ eGFR $\left(\mathrm{mL} / \mathrm{min} / 1.73 \mathrm{~m}^{2}\right)$} \\
\hline \multicolumn{5}{|l|}{$<10$} \\
\hline$\geq 10$ & $0.35(0.21,0.56)$ & $<0.001$ & $0.46(0.28,0.76)$ & $<0.01$ \\
\hline
\end{tabular}

Crude HR: represented relative hazard ratio; adjusted HR: represented adjusted hazard ratio adjusted for $\mathrm{CHM}$ use, primary disease, $\mathrm{Hb}$, $\mathrm{K}, \mathrm{CO}_{2} \mathrm{CP}$, and eGFR in the multivariate Cox regression analysis. CKD, chronic kidney disease; HR, hazard ratio; $\mathrm{Cl}$, confidence interval; $\mathrm{CHM}$, Chinese herbal medicine; $\mathrm{BMI}$, body mass index; $\mathrm{ALB}$, albumin; $\mathrm{Hb}$, hemoglobin; $\mathrm{K}$, serum potassium; $\mathrm{CO}_{2} \mathrm{CP} \mathrm{carbon}$ dioxide combining power; eGFR, estimated glomerular filtration rate. 


\begin{tabular}{|c|c|c|c|}
\hline Items & CHM group $(n=747)$ & Non-CHM group $(n=67)$ & $P$ \\
\hline Hyperkalemia & 160 & 2 & 0.59 \\
\hline Infection (unknown reasons) & 4 & 1 & 0.08 \\
\hline Hypocalcemia & 1 & 0 & 1.00 \\
\hline Constipation & 1 & 0 & 1.00 \\
\hline Metabolic acidosis & 9 & 0 & 1.00 \\
\hline Zoster & 2 & 0 & 1.00 \\
\hline Cholecystitis & 1 & 0 & 1.00 \\
\hline Hypokalemia & 3 & 0 & 1.00 \\
\hline Electrolyte disturbances & 1 & 0 & 1.00 \\
\hline Leukopenia & 1 & 0 & 1.00 \\
\hline Multiple lacunar infarction & 1 & 0 & 1.00 \\
\hline Fever & 2 & 0 & 1.00 \\
\hline Pulmonary infection & 2 & 0 & 1.00 \\
\hline Gastric distention & 1 & 0 & 1.00 \\
\hline Abdominal pain & 2 & 0 & 1.00 \\
\hline Ruptured abdominal aorta aneurysm & 1 & 0 & 1.00 \\
\hline Allergic dermatitis & 1 & 0 & 1.00 \\
\hline Fracture & 1 & 0 & 1.00 \\
\hline Chronic renal failure worsens & 6 & 0 & 1.00 \\
\hline Urinary tract infection & 1 & 0 & 1.00 \\
\hline Bladder cancer & 1 & 0 & 1.00 \\
\hline Upper respiratory tract infection & 24 & 0 & 1.00 \\
\hline Eczema & 1 & 0 & 1.00 \\
\hline Death & 1 & 0 & 1.00 \\
\hline Gout attack & 1 & 0 & 1.00 \\
\hline Dizziness & 1 & 0 & 1.00 \\
\hline Epilepsy & 1 & 0 & 1.00 \\
\hline Painless hematuria & 1 & 0 & 1.00 \\
\hline
\end{tabular}

CHM, Chinese herbal medicine.

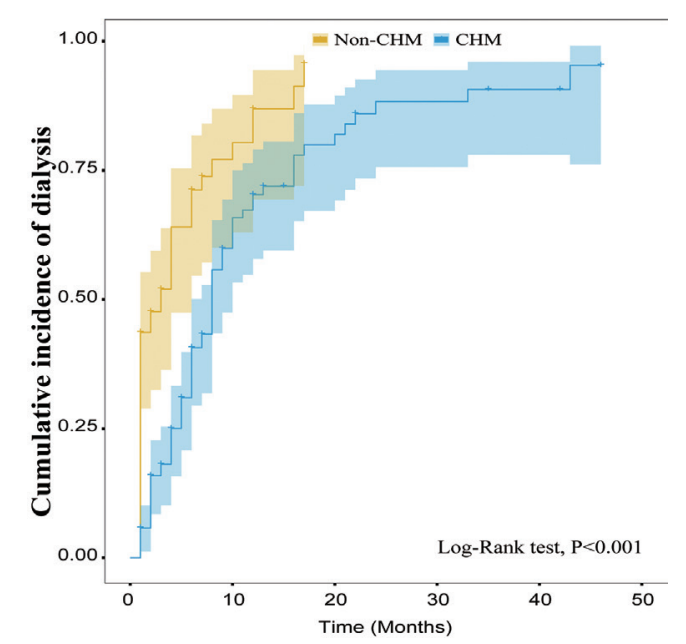

Figure S1 Cumulative incidence rate of dialysis among patients with chronic kidney disease (CKD) stage 5 in the Chinese herbal medicine (CHM) group and the non-CHM group (after 1:2 matching with a caliper of 0.05 ). 